\title{
Microscopic formulation of a lattice-defect model
}

\author{
A. Holz* \\ Instituto de Física, Universidade Federal do Rio Grande do Sul, 90000 Porto Alegre, RS, Brasil \\ (Received 20 April 1979; revised manuscript received 21 August 1979)
}

\begin{abstract}
A microscopic construction method of lattice defects which can be represented by dislocation configurations in simple-cubic lattices and for interaction by central forces is given. In contrast to standard theories, where dislocations are introduced via topological operations (Burgers circuit), the author starts from a microscopic ad hoc Hamiltonian which is suitable only for central-force interacting systems. The various sectors of this microscopic Hamiltonian are associated with dislocation configurations. A detailed discussion of the stability and symmetry properties of the microscopic Hamiltonian is given. Possible extensions of the theory to non-central-force interacting systems are pointed out.
\end{abstract}

\section{INTRODUCTION}

The theory of structural defects in crystal lattices has been developed by many workers and the main theoretical concepts and literature references can be found in Refs. 1-4. In the following work a microscopic formulation of the theory will be attempted which differs from most of the theories presented in Refs. 1-4 in one basic point in that we deduce the defects directly from a basic lattice Hamiltonian of the system.

The basic lattice Hamiltonian is a generalized rotator model. One knows already that the topological defects of the planar rotator model are vortex loops with discrete current strengths which interact via the Biot-Savard law with opposite sign. Accordingly a generalized rotator model with a conserved vector current can be expected to model dislocation theory in solids. Such an analogy with magnetostatics, in particular with respect to Biot-Savard's law, was first pointed out by Kröner. ${ }^{1}$

In the standard theories of dislocations, dislocations are introduced into the lattice via topological operations, i.e., the Burgers circuit, and phenomenological expressions which are based on continuum elasticity theory. There also exist, however, examples of calculations of lattice defects like vacancies and interstitials based on a discrete-lattice computational approach. ${ }^{2,5}$ Furthermore Kochendörfer and Seeger ${ }^{6}$ treated the moving dislocation from a discrete-lattice point of view. The spirit of the following approach differs, however, from such formalisms and is closer to the theory of topological defects as it is presently in vogue in the theory of Ising-like models in statistical mechanics (see, e.g., Holz $\mathrm{H}^{7}$ and references therein). The idea there is to start out from a lattice Hamiltonian which describes the system properly in all its possible configurations. The defect states represent then the various sectors of the phase space of that Hamiltonian. This approach should therefore also allow the description of the various possible phases of such a "crys talline" lattice system, e.g., its liquid phase. We have to admit, however, that so far we have not been able to succeed completely in such an ambitious enterprise. Nevertheless it is hoped that the present approach, when suitably modified will lead to some progress in this field.

The plan of the paper is the following. In Sec. II the basic Hamiltonian of the present theory will be presented and a discussion of its stability and symmetry conditions will be given. In Sec. III a derivation of a dislocation theory based on the present concepts and starting from a simple intuitive expression for the Hamiltonian will be given and in Sec. IV a discussion of the isotropic case will be presented. The computational details of Secs. III and IV are worked out in the Appendix. In Sec. $\mathrm{V}$ a discussion of the ideas developed in this work will be given. It should be pointed out that the methods to be presented apply to dimensions $D \geqslant 2$ but will only be worked out for $D=3$.

\section{BASIC HAMILTONIAN, STABILITY AND SYMMETRY}

For a classical lattice without any defects the lattice potential can be represented as a Taylor expansion around a periodic equilibrium configuration in the form ${ }^{8}$

$$
\phi\left(\left\{\overrightarrow{\mathrm{R}}^{\overrightarrow{\mathrm{n}}}\right\}\right)=\phi_{0}+\frac{1}{2} \sum_{\substack{\mathrm{m}, \overrightarrow{\mathrm{n}} \\ i, k}} \Phi_{i, k}^{\overrightarrow{\mathrm{m}}, \overrightarrow{\mathrm{n}}} S_{i}^{\overrightarrow{\mathrm{m}}} S_{k}^{\overrightarrow{\mathrm{n}}}+\cdots .
$$

Here for the sake of simplicity we study a simplecubic lattice with $N$ lattice constituents at the equilibrium sites

$$
\left\{\overrightarrow{\mathrm{R}}_{0}^{\overrightarrow{\mathrm{n}}}\right\} \equiv\left(\overrightarrow{\mathrm{R}}_{0}^{1}, \overrightarrow{\mathrm{R}}_{0}^{2}, \ldots, \overrightarrow{\mathrm{R}}_{0}^{N}\right)
$$

and denote deviations from $\vec{R}_{0}^{\vec{n}}$ by $\vec{S}^{\vec{n}}$, i.e., $\vec{R}^{\vec{n}}$ $=\overrightarrow{\mathbf{R}}_{0}^{\vec{n}}+\overrightarrow{\mathrm{S}}^{\overrightarrow{\mathrm{n}}}$. $\Phi_{i, k}^{\overrightarrow{\mathrm{m}} \overrightarrow{\mathrm{n}}}$ is a coupling constant matrix and 
$i=1,2, \ldots, D$ runs over the $D$ Cartesian coordinates of the lattice constituents. From homogeneity we take

$$
\Phi_{i, k}^{\overrightarrow{\mathrm{m}}, \overrightarrow{\mathrm{n}}}=\Phi_{i, k}^{\overrightarrow{\mathrm{m}}-\overrightarrow{\mathrm{n}}}=\Phi_{i, k}^{\overrightarrow{\mathrm{n}}-\overrightarrow{\mathrm{m}}}
$$

and from the cubic symmetry

$$
\Phi_{i, k}^{\overrightarrow{\mathrm{h}}}=\Phi_{k, i}^{-\overrightarrow{\mathrm{h}}} \text {. }
$$

Equation (1) is the harmonic approximation of lattice theory and the neglected anharmonic terms will be considered later.

If we introduce the quantity

$$
S_{i}^{\overrightarrow{\mathrm{m}}-\overrightarrow{\mathrm{n}}} \equiv S_{i}^{\overrightarrow{\mathrm{m}}}-S_{i}^{\overrightarrow{\mathrm{n}}}
$$

then Eq. (1) can be written in the form

$$
\begin{aligned}
\Phi(\{\overrightarrow{\mathrm{R}} \overrightarrow{\mathrm{n}}\})= & \Phi_{0}-\frac{1}{4} \sum_{\substack{\mathrm{m}, \overrightarrow{\mathrm{n}} \\
i, k}} \Phi_{i, k}^{\overrightarrow{\mathrm{m}}-\overrightarrow{\mathrm{n}}} S_{i}^{\overrightarrow{\mathrm{m}}-\overrightarrow{\mathrm{n}}} S_{k}^{\overrightarrow{\mathrm{m}}-\overrightarrow{\mathrm{n}}} \\
& +\frac{1}{4} \sum_{\substack{\overrightarrow{\mathrm{m}}, \overrightarrow{\mathrm{n}} \\
i, k}} \Phi_{i, k}^{\overrightarrow{\mathrm{m}}-\overrightarrow{\mathrm{n}}}\left(S_{i}^{\overrightarrow{\mathrm{m}}} S_{k}^{\overrightarrow{\mathrm{m}}}+S_{i}^{\overrightarrow{\mathrm{n}}} S_{k}^{\overrightarrow{\mathrm{n}}}\right) .
\end{aligned}
$$

Because of the relation [see Eq. (36.2) in Ref. 8]

$$
\sum_{\overrightarrow{\mathrm{h}}=0} \Phi_{i, k}^{\overrightarrow{\mathrm{h}}}=0 \text {, }
$$

the third term of Eq. $\left(1^{\prime}\right)$ vanishes. Equation ( $\left.1^{\prime}\right)$ forms the starting point of the following developments.

Assume now that a defect like a dislocation is introduced into the lattice. The only simple way to do that is to produce an internal cut surface of dimension $D-1$ and displace the two sides of the cut surface. The displacement of the atoms along the cut surface is done such that the displacement vectors $\left\{\vec{b}^{\overrightarrow{\mathrm{m}}, \overrightarrow{\mathrm{n}}}\right\}$ are multiples of lattice vectors. Consider next two lattice constituents at sites $\vec{R}_{0}^{\mathrm{n}}$ and $\vec{R}_{0}^{\overrightarrow{\mathrm{m}}}$, respectively. Changing their relative displacement by

$$
\vec{S}^{\vec{n}-\vec{m}} \rightarrow \vec{S}^{\vec{n}-\vec{m}}+\vec{b}^{n}, \vec{m}
$$

puts them, because, of the periodicity of the lattice, into an environment which may exert on them the same forces as for $\vec{b} \vec{n}, \vec{m}=0$. However, in order that this is the case other lattice constituents have also to be moved in order to make the displacement compatible with the lattice. One may expect therefore that the Hamiltonian for a certain defect structure characterized by a set of vectors $\{\vec{b} \vec{n}, \vec{m}\}$ is in the "harmonic" approximation of the form

$$
\begin{aligned}
& \Phi^{h}\left(\left\{\overrightarrow{\mathrm{R}_{0}^{\mathrm{n}}}\right\},\left\{\overrightarrow{\mathrm{b}^{\mathrm{n}}, \overrightarrow{\mathrm{m}}}\right\}\right) \\
& =\Phi_{0}-\frac{1}{4} \sum_{\substack{\overrightarrow{\mathrm{m}}, \overrightarrow{\mathrm{n}} \\
i, k}} \Phi_{i, k}^{\overrightarrow{\mathrm{m}}-\overrightarrow{\mathrm{n}}}\left(\vec{S}_{i}^{\overrightarrow{\mathrm{m}}-\overrightarrow{\mathrm{n}}}-b_{i}^{\overrightarrow{\mathrm{m}}, \overrightarrow{\mathrm{n}}}\right)\left(\overrightarrow{\mathrm{S}}_{k}^{\overrightarrow{\mathrm{m}}-\overrightarrow{\mathrm{n}}}-b_{k}^{\overrightarrow{\mathrm{m}}, \overrightarrow{\mathrm{n}}}\right) .
\end{aligned}
$$

It follows from the procedure of constructing the vectors $\overrightarrow{\mathrm{b}}^{\overrightarrow{\mathrm{m}}, \overrightarrow{\mathrm{n}}}$ that

$$
\overrightarrow{\mathrm{b}}^{\overrightarrow{\mathrm{m}}, \overrightarrow{\mathrm{a}}}=-\overrightarrow{\mathrm{b}}^{\mathrm{n}}, \overrightarrow{\mathrm{m}}
$$

has to hold.

In order that this expression leads to sensible results the coupling constant matrix $\left\{\Phi_{i, j}^{\overrightarrow{\mathrm{A}}, \overrightarrow{\mathrm{m}}}\right\}$ has to fulfill two conditions: First, $\left\{+\Phi_{i, j}^{\vec{n}, \vec{m}}\right\}$ necessarily has to be positive definite in the space of the dis placement variables $\left\{\overrightarrow{\mathrm{S}^{\mathrm{n}}}\right\}$. Second, $\left\{-\Phi_{i, j}^{\overrightarrow{\mathrm{h}}}\right\}_{\overrightarrow{\mathrm{h}}}$ has to be positive definite for fixed $\vec{h} \neq 0$ in the space of the difference variables $\left\{\overrightarrow{S^{\mathrm{h}}}\right\}$ which are defined by Eq. (4). If the latter condition is not fulfilled then Eq. (7) may be unstable against defect production even if it is stable with respect to phonon excitations. If $\left\{-\Phi_{i, j}^{\overrightarrow{\mathrm{h}}}\right\}_{\overrightarrow{\mathrm{h}}}$ is positive definite, then

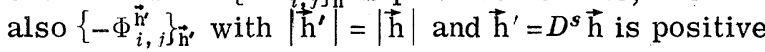
definite because both are related via a symmetry operation $D^{s}$ which will not change the eigenvalues of $\left\{-\Phi_{i, j}^{\overrightarrow{\mathrm{h}}}\right\} \overrightarrow{\mathrm{h}}$. The positive definiteness of $\left\{\Phi_{i, \vec{j}}^{\overrightarrow{\mathrm{n}}, \overrightarrow{\mathrm{m}}}\right\}$ can always be imposed by means of the terms $\Phi_{i, j}^{n, n}$ which do not influence the elastic properties. The positive definiteness of each $\left\{-\Phi_{i, j}^{\overrightarrow{\mathrm{h}}}\right\}_{\mathrm{h}}$ matrix implies that the inverse propagator defined in Eq. (20) is always positive definite and this leads to a stable phonon spectrum. Although the second condition is sufficient for stability we are not certain that it is also necessary. The final results of the present theory will involve in addition the coefficients [see Eq. (58.4a) of Ref. 8]

$$
\widetilde{C}_{i k, n m}=-\frac{1}{2} \sum_{\overrightarrow{\mathrm{h}}} \Phi_{i, k}^{\overrightarrow{\mathrm{h}}} h_{n} h_{m}
$$

which ultimately will determine the stability of the system. We will return to this problem later.

Let us point out that if the displacement of lattice constituents along the cut surface could be done in a completely compatible way then Eq. (7) should be transformable into Eq. (1'). That this is in fact the case for closed cut surfaces and infinitely extended cut surfaces which are closed over the periodic boundary conditions will be shown later. For finite and open cut surfaces the displacements described by $\left\{\vec{b}^{\vec{m}}, \vec{n}\right\}$ are not compatible. Accordingly $\overrightarrow{\mathrm{S}}^{\overrightarrow{\mathrm{n}}-\overrightarrow{\mathrm{m}}}=\overrightarrow{\mathrm{b}}^{\mathrm{i}}, \overrightarrow{\mathrm{m}}$ does not represent a solution to Eq. (7) and internal strain arises in the system. A more detailed explanation of the use of Eq. (7) will be given later. Note that Eq. (7) is in a covariant form, i.e., it preserves its shape when transformed to a primed coordinate system. Although Eq. (7) is obviously invariant against any translation it is not necessarily invariant against any rotation of the lattice constituents. Consider first a symmetry operation $D^{s}$ which transforms lattice constituents into equivalent lattice constituents

$$
D^{s} \overrightarrow{\mathrm{R}_{0}^{\overrightarrow{\mathrm{n}}}}=\overrightarrow{\mathrm{R}}_{0}^{\vec{n}^{\prime}} .
$$


If $\vec{b} \vec{n}, \vec{m}$ is defined with respect to the actual lattice sites $\overrightarrow{\mathrm{R}}^{\mathbb{n}}$ then $D^{s}$ also transforms the set of vectors $\vec{b}^{\vec{n}, \vec{m}}$, i.e.,

$$
D^{s} \overrightarrow{\mathrm{b}^{\mathrm{n}}, \overrightarrow{\mathrm{m}}}=\overrightarrow{\mathrm{b}}^{\overrightarrow{\mathrm{n}}^{\prime}, \overrightarrow{\mathrm{m}}^{\prime}}
$$

Invariance of Eq. (7) with respect to $D^{s}$ requires

$$
\Phi\left(\left\{\overrightarrow{\mathrm{b}}^{\overrightarrow{\mathrm{n}}^{\prime}, \overrightarrow{\mathrm{m}}^{\prime}}\right\}\right)=\Phi(\{\overrightarrow{\mathrm{b}} \overrightarrow{\mathrm{n}}, \overrightarrow{\mathrm{m}}\})
$$

and this leads to

$$
\sum_{i^{\prime}, k^{\prime}} \Phi_{i^{\prime}, k^{\prime}}^{\overrightarrow{\mathrm{D}^{\prime}}, \overrightarrow{\mathrm{m}^{\prime}}} D_{i^{\prime} i}^{s} D_{k^{\prime} k}^{s}=\Phi_{i, k}^{\overrightarrow{\mathrm{n}}, \overrightarrow{\mathrm{m}}}
$$

This set of equations represents the usual symmetry properties of the coupling constants [see Eq. (36.7) Leibfried ${ }^{8}$. Consider next a general infinitesimal rotation $\Omega-I$ of the lattice, where $I$ is the unit matrix. Rotational invariance then requires that

$$
\Phi\left(\left\{\Omega\left(\vec{R}^{\vec{n}}\right)\right\}\right)=\Phi\left(\left\{\vec{R}^{\vec{n}}\right\}\right)
$$

holds. Applied to the Hamiltonian of a certain sector of the phase space characterized by the set $\{\vec{b} \vec{n}, \vec{m}\}$ the above condition reads

$$
\Phi\left(\left\{\Omega\left(\overrightarrow{\mathrm{R}}_{0}^{n}+\overrightarrow{\mathrm{S}}^{n}\right)\right\},\left\{\overrightarrow{\mathrm{b}^{\mathrm{n}}, \overrightarrow{\mathrm{m}}}\right\}\right)=\Phi\left(\left\{\overrightarrow{\mathrm{R}}_{0}^{n}+\overrightarrow{\mathrm{S}}^{n}\right\},\{\overrightarrow{\mathrm{b}} \overrightarrow{\mathrm{n}}, \overrightarrow{\mathrm{m}}\}\right),
$$

where it should be noted that the set $\{\vec{b} \vec{n}, \vec{m}\}$ is not subject to the rotation because it is a passive set of variables and refers only to the sectors of the phase space. For an infinitesimal rotation the left-hand side of Eq. (14) can be expanded in terms of $\Omega-I$. Keeping only up to quadratic terms in the small quantities $\Omega-I$, and $\overrightarrow{\mathrm{S}}^{\vec{n}}$ the condition $\mathrm{Eq}$. (14) requires

$$
\begin{aligned}
& \frac{\partial^{\alpha}}{\partial \Omega^{\alpha}} \Phi\left(\left\{\Omega\left(\overrightarrow{\mathrm{R}}_{0}^{\overrightarrow{\mathrm{n}}}+\overrightarrow{\mathrm{S}}^{\overrightarrow{\mathrm{n}}}\right)\right\},\left\{\overrightarrow{\mathrm{b}}^{\overrightarrow{\mathrm{n}}, \overrightarrow{\mathrm{m}}}\right\}\right) \underset{\Omega=I}{=0,} \\
& \alpha=1,2 .
\end{aligned}
$$

A necessary condition that Eq. (15) is satisfied is [see Eq. (36.3) Ref. 8]

$$
\sum_{\overrightarrow{\mathrm{n}}} \Phi_{i, k}^{\overrightarrow{\mathrm{m}}, \overrightarrow{\mathrm{n}}} R_{0, l}^{\overrightarrow{\mathrm{n}}}=\sum_{\overrightarrow{\mathrm{n}}} \Phi_{i, l}^{\overrightarrow{\mathrm{m}}, \overrightarrow{\mathrm{n}}} R_{0, k}^{\overrightarrow{\mathrm{n}}} .
$$

This is the usual condition for invariance under infinitesimal rotations in the harmonic approximation and without defects. That Eq. (16) is also a sufficient condition in the presence of defects or, say, for the harmonic approximation of the defect Hamiltonian Eq. (7) cannot be said without computation of Eq. (15). In Sec. III it will be shown that the problem defined by Eqs. (7) and (8) can be transformed into an expression of the form

$$
H=H_{\mathrm{ph}}+H_{\mathrm{def}}\left(\left\{\overrightarrow{\mathbf{b}}^{\sigma}, C_{\sigma}\right\}\right),
$$

where $\overrightarrow{\mathrm{b}}^{\sigma}$ and $C_{\sigma}$ represent the Burgers vector and associated contour, respectively, and $\left\{\vec{b}^{\sigma}, C_{\sigma}\right\}$ is a set of such pairs which defines the total defect structure. The first term of Eq. (17) represents a phonon Hamiltonian and the second term represents the interaction between the defects. If a similar equation is derived from the left-hand side of Eq. (14) one obtains instead of Eq. (17)

$$
H=H_{\mathrm{ph}}+H_{\text {def }}\left(\left\{\overrightarrow{\mathrm{b}}^{\sigma}, C_{\sigma}\right\}, \Omega-I\right)+\cdots,
$$

where third-order terms in the small quantities $\Omega-I$, and $\overrightarrow{\mathrm{S}}^{n}$ have been dropped, and where Eq. (16) has already been taken into account. The invariance condition

$$
H_{d e f}\left(\left\{\overrightarrow{\mathrm{b}}^{\sigma}, C_{\sigma}\right\}, \Omega-I\right)=H_{\text {def }}\left(\left\{\overrightarrow{\mathrm{b}}^{\sigma}, C_{\sigma}\right\}\right)
$$

leads then to additional conditions for the coupling constant matrix besides those given by Eq. (16). On the other hand, if one works with a given coupling-constant matrix $\left\{\Phi_{i, k}^{\overrightarrow{\mathrm{n}}, \overrightarrow{\mathrm{m}}}\right\}$, then Eq. (7) has to be supplemented by additional terms in order that Eq. (18) is satisfied. In particular it is shown in Sec. III that only for central two-body forces the displacement fields computed by means of the present theory agree with those of the standard theory. Accordingly for noncentral forces Eq. (7) has to be modified. Such modifications will be discussed at the end of Sec. III and in Sec. V.

\section{MICROSCOPIC FORMULATION OF "DISLOCATION" THEORY}

In order to find the equilibrium solutions to Eq. (7) for fixed $\left\{\vec{b}^{\vec{n}-m}\right\}$ we perform a Fourier transformation and diagonalize by means of trans lation of variables. Equation (7) will be written in the form

$$
\begin{aligned}
\Phi\left(\left\{\overrightarrow{\mathrm{b}^{\mathrm{m}}, \overrightarrow{\mathrm{n}}}\right\}\right)= & \Phi_{0}-\frac{1}{4} \sum_{\substack{\overrightarrow{\mathrm{m}}, \overrightarrow{\mathrm{n}} \\
i, k}} \Phi_{i, k}^{\overrightarrow{\mathrm{m}}-\overrightarrow{\mathrm{n}}} S_{i}^{\overrightarrow{\mathrm{m}}-\overrightarrow{\mathrm{n}}} S_{k}^{\overrightarrow{\mathrm{m}}-\overrightarrow{\mathrm{n}}}+\sum_{\substack{\overrightarrow{\mathrm{m}}, \overrightarrow{\mathrm{n}} \\
i, k}} \Phi_{i, k}^{\overrightarrow{\mathrm{m}}-\overrightarrow{\mathrm{n}}} b_{i}^{\overrightarrow{\mathrm{m}}, \overrightarrow{\mathrm{n}}} S_{k}^{\overrightarrow{\mathrm{m}}} \\
& -\frac{1}{4} \sum_{\substack{\overrightarrow{\mathrm{m}}, \overrightarrow{\mathrm{n}} \\
i, k}} \Phi_{\substack{\overrightarrow{\mathrm{m}}-\vec{n} \\
\mathrm{~m}}}^{\overrightarrow{\mathrm{m}}} b_{i}^{\overrightarrow{\mathrm{m}}, \overrightarrow{\mathrm{n}}} b_{k}^{\overrightarrow{\mathrm{m}}, \overrightarrow{\mathrm{n}}} .
\end{aligned}
$$

Introducing the Fourier transforms

$$
\begin{aligned}
& S_{k}^{\overrightarrow{\mathrm{m}}}=\frac{1}{\sqrt{N}} \sum_{\overrightarrow{\mathrm{q}}} S_{\overrightarrow{\mathrm{q}}, k} e^{-i \overrightarrow{\mathrm{q}} \cdot \overrightarrow{\mathrm{R}}_{0}^{\overrightarrow{\mathrm{m}}}}, \\
& S_{i}^{\overrightarrow{\mathrm{m}}-\overrightarrow{\mathrm{n}}}=\frac{1}{\sqrt{N}} \sum_{\overrightarrow{\mathrm{q}}} S_{\overrightarrow{\mathrm{q}}, i} e^{-i \overrightarrow{\mathrm{q}} \cdot \overrightarrow{\mathrm{R}}_{0}^{\overrightarrow{\mathrm{m}}}}\left(1-e^{-\overrightarrow{\mathrm{q}} \cdot \overrightarrow{\mathrm{h}}}\right)
\end{aligned}
$$

into Eq. (7') leads to

$$
\begin{aligned}
\Phi(\{\overrightarrow{\mathrm{b}} \overrightarrow{\mathrm{m}}, \overrightarrow{\mathrm{n}}\})= & \Phi_{0}-\frac{1}{2} \sum_{\overrightarrow{\mathrm{q}}} \sum_{\substack{\overrightarrow{\mathrm{h}} \\
i, k}} \Phi_{i, k}^{\overrightarrow{\mathrm{n}}} S_{\overrightarrow{\mathrm{q}}, i} S_{-\overrightarrow{\mathrm{q}}, k}(1-\cos \overrightarrow{\mathrm{q}} \cdot \overrightarrow{\mathrm{h}}) \\
& +\frac{1}{\sqrt{N}} \sum_{\overrightarrow{\mathrm{q}}} \sum_{\overrightarrow{\mathrm{m}}, \overrightarrow{\mathrm{n}}} S_{\overrightarrow{\mathrm{q}}, k} \Phi_{i, k}^{\overrightarrow{\mathrm{m}}-\overrightarrow{\mathrm{n}}} b_{i}^{\overrightarrow{\mathrm{m}}-\overrightarrow{\mathrm{n}}} e^{-i \overrightarrow{\mathrm{a}} \cdot \overrightarrow{\mathrm{r}}_{0}^{\overrightarrow{\mathrm{m}}}} \\
& -\frac{1}{4} \sum_{\substack{\overrightarrow{\mathrm{m}}, \overrightarrow{\mathrm{n}} \\
i, k}} \Phi_{i, k}^{\overrightarrow{\mathrm{m}}-\overrightarrow{\mathrm{n}}} b_{i}^{\overrightarrow{\mathrm{m}}, \overrightarrow{\mathrm{n}}} b_{k}^{\overrightarrow{\mathrm{m}}, \overrightarrow{\mathrm{n}}},
\end{aligned}
$$


where $\vec{q}$ runs over the first Brillouin zone. Also introducing the notation

$$
\Phi_{i, k}(\overrightarrow{\mathrm{q}}) \equiv-\sum_{\overrightarrow{\mathrm{h}}} \Phi_{i, k}^{\overrightarrow{\mathrm{h}}}(1-\cos \overrightarrow{\mathrm{q}} \cdot \overrightarrow{\mathrm{h}})
$$

and diagonalizing Eq. (19) by means of the shift operation

$$
S_{\overrightarrow{\mathrm{q}}, k}=S_{\overrightarrow{\mathrm{q}}, k}^{\prime}+p_{\overrightarrow{\mathrm{q}}, k},
$$

where

$$
p_{\overrightarrow{\mathrm{q}}, j}=-\frac{1}{\sqrt{N}} \Phi_{j, k}^{-1}(\overrightarrow{\mathrm{q}}) \sum_{\overrightarrow{\mathrm{m}}, \overrightarrow{\mathrm{n}}} \Phi_{k, i}^{\overrightarrow{\mathrm{m}}-\overrightarrow{\mathrm{n}}} b_{i}^{\overrightarrow{\mathrm{m}}, \overrightarrow{\mathrm{n}}} e^{i \overrightarrow{\mathrm{q}} \cdot \overrightarrow{\mathrm{R}}_{0}^{\overrightarrow{\mathrm{m}}}},
$$

one obtains

$$
\begin{aligned}
& \Phi\left(\left\{\overrightarrow{\mathrm{b}}^{\overrightarrow{\mathrm{m}}, \overrightarrow{\mathrm{n}}}\right\}\right)=\Phi_{0}+\frac{1}{2} \sum_{\overrightarrow{\mathrm{q}}} S_{\overrightarrow{\mathrm{q}}, i}^{\prime} \Phi_{i, k}(\overrightarrow{\mathrm{q}}) \overrightarrow{\mathrm{S}}_{-\overrightarrow{\mathrm{q}}, k}^{\prime}-\frac{1}{2 N} \sum_{\overrightarrow{\mathrm{q}}}\left(\sum_{\overrightarrow{\mathrm{m}}, \overrightarrow{\mathrm{n}}} \Phi_{j, s}^{\overrightarrow{\mathrm{m}}-\overrightarrow{\mathrm{n}}} b_{s}^{\overrightarrow{\mathrm{m}}, \overrightarrow{\mathrm{n}}} e^{i \overrightarrow{\mathrm{q}} \cdot \overrightarrow{\mathrm{a}}_{0}^{\overrightarrow{\mathrm{m}}}}\right)_{\Phi_{j, i^{\prime}}^{-1}}(\overrightarrow{\mathrm{q}}) \\
& \times\left(\sum_{\overrightarrow{\mathrm{m}}^{\prime}, \overrightarrow{\mathrm{n}}^{\prime}} \Phi_{i^{\prime}, s^{\prime}}^{\overrightarrow{\mathrm{m}}^{\prime}-\overrightarrow{\mathrm{n}}^{\prime}} b_{s^{\prime}}^{\overrightarrow{\mathrm{m}}^{\prime}, \overrightarrow{\mathrm{n}}^{\prime}} e^{-i \overrightarrow{\mathrm{q}} \circ \overrightarrow{\mathrm{R}}} \overrightarrow{0}_{0}^{\overrightarrow{\mathrm{m}}^{\prime}}\right)-\frac{1}{4} \sum_{\overrightarrow{\mathrm{m}}, \overrightarrow{\mathrm{n}}} \Phi_{i, k}^{\overrightarrow{\mathrm{m}}-\overrightarrow{\mathrm{n}}} b_{i}^{\overrightarrow{\mathrm{m}}, \overrightarrow{\mathrm{n}}} b_{k}^{\overrightarrow{\mathrm{m}}, \overrightarrow{\mathrm{n}}} .
\end{aligned}
$$

Here and in the following Einstein summation convention will be used for subindices. The second term of Eq. (23) represents the phonon excitations in the defect state and the following terms represent the defect interaction Hamiltonian $\Phi^{\text {int }}\left(\left\{b^{\overrightarrow{\mathrm{m}}, \overrightarrow{\mathrm{n}}}\right\}\right)$. Further manipulations to transform Eq. (23) are given in the Appendix and lead to

$$
\begin{aligned}
& \Phi^{\mathrm{int}}\left(\left\{\overrightarrow{\mathrm{b}^{\mathrm{n}}, \overrightarrow{\mathrm{m}}}\right\}\right)=-\frac{1}{2 N} \sum_{\overrightarrow{\mathrm{q}}} \sum_{\sigma, \sigma^{\mathrm{j}}}\left[\tilde{C}_{j s, \beta \sigma} b_{s}^{\sigma} \int_{C_{\sigma}} d r_{\alpha}^{\sigma} \epsilon_{\alpha \beta \gamma}\left(q_{\gamma}^{0} q_{\sigma}^{0} e^{i \overrightarrow{\mathrm{q}} \cdot \overrightarrow{\mathrm{r}}^{\sigma}}\right) \Phi_{j, i^{\prime}}^{-1}(\overrightarrow{\mathrm{q}})\right. \\
& \times\left(\tilde{C}_{i^{\prime} s^{\prime}, \beta^{\prime} \sigma^{\prime}} b_{s^{\prime}}^{\sigma^{\prime}} \int_{C_{\sigma^{\prime}}} d r_{\alpha^{\prime}}^{\sigma^{\prime}, \epsilon_{\alpha^{\prime} \beta^{\prime} \gamma^{\prime}}} q_{\gamma^{\prime}}^{0} q_{\delta^{\prime}}^{0} e^{-i \vec{q} \cdot \vec{r}^{\sigma^{\prime}}}\right) \\
& \left.-\tilde{C}_{s s^{\prime}, \beta \beta^{\prime}} b_{s}^{\sigma} b_{s^{\prime}}^{\sigma^{\prime}} \int_{C_{\sigma}} \int_{C_{\sigma^{\prime}}} d r_{\alpha}^{\sigma} d r_{\alpha^{\prime}}^{\sigma^{\prime}} \epsilon_{\alpha_{\beta} \gamma} \epsilon_{\alpha^{\prime} \beta^{\prime} \gamma^{\prime}} \frac{q_{\gamma}^{0} q_{\gamma}^{0}, e^{i \overrightarrow{\mathrm{q}\left(\overrightarrow{\mathrm{r}}^{\sigma}-\overrightarrow{\mathrm{r}}^{\sigma^{\prime}}\right)}}}{q^{2}}\right],
\end{aligned}
$$

where $\overrightarrow{\mathrm{q}}^{0}=\overrightarrow{\mathrm{q}} /|\overrightarrow{\mathrm{q}}|$ and $\epsilon_{\alpha_{\beta \gamma}}$ is the Levi-Civita tensor. Note that the Burgers vector $\vec{b}^{\sigma}$ is defined with respect to the cut surface $\Sigma_{\sigma}$ and therefore is conserved along the contour $C_{\sigma}$ bordering $\Sigma_{\sigma}$. Equation (24) has been derived under the assumption that the contours $C_{\sigma}$ are well separated from each other. Suppose now that $n$ contours $C_{\sigma}, \sigma$ $=1,2, \ldots, n$ coalesce along the path $C^{\prime}$. From Eq. (24) it follows that $\Phi^{\text {int }}$ is completely symmetrical in all $C_{\sigma}$; i.e., it is a bilinear form of expressions of the type $\tilde{C} \cdots b_{\bullet}^{\sigma} \int_{c_{\sigma}} d r^{\sigma}$, where the points stand for subindices.: This implies that for

$$
C^{\prime} \cap \prod_{\sigma=1}^{n} c_{\sigma} \neq \emptyset
$$

$\sum_{\sigma=1}^{n} \vec{b}^{\sigma}$ enters $\Phi^{\text {int }}$. Because $\vec{b}^{\sigma}$ is conserved along each closed contour, coalescence of contours. implies that the Burgers vector is conserved at branch points of the contours and furthermore that the interaction law envolves always the resulting Burgers vector. Accordingly the present Burgers vectors follow the same conservation laws as do the standard Burgers vectors used in the theory of plasticity and lattice defects. Note that for the case of infinitely extended cut sur- faces which are closed over the periodic boundary condition $\Phi^{\text {int }}$ vanishes.

Next we determine the Fourier transforms $p_{\overrightarrow{\mathbf{q}}, j}$ defined by Eq. (22). Using the same approximations under which Eq. (A $\left.7^{\prime}\right)$ of the Appendix is derived Eq. (22) can be written in the form

$$
\begin{aligned}
p_{-\overrightarrow{\mathrm{q}}, j}=-\frac{1}{\sqrt{N}} \Phi_{j, k}^{-1}(\overrightarrow{\mathrm{q}}) \frac{1}{2} \sum_{\sigma, \overrightarrow{\mathrm{h}}} \int_{\Sigma_{\sigma}} & \left(d^{2} \overrightarrow{\mathrm{r}}^{\sigma} \cdot \overrightarrow{\mathrm{h}}\right) \Phi_{k, i}^{\overrightarrow{\mathrm{h}}} \\
& \times b_{i}^{\sigma}(i \overrightarrow{\mathrm{q}} \cdot \overrightarrow{\mathrm{h}}) e^{-i \overrightarrow{\mathrm{q}} \cdot \overrightarrow{\mathrm{r}}^{\sigma}} .
\end{aligned}
$$

Using Eq. (9), allows to write Eq. (20) in the longwavelength approximation in the form

$$
\Phi_{i, k}(\overrightarrow{\mathrm{q}})=\tilde{C}_{i k, s s^{\prime}} q_{s} q_{s^{\prime}} \text {. }
$$

Going over to Hook's tensor by means of the relation [see Eq. (58.6) of Leibfried ${ }^{8}$ ]

$$
\tilde{C}_{i k, s s^{\prime}}=\frac{1}{2}\left(C_{i s, k s^{\prime}}+C_{i s^{\prime}, k s}\right),
$$

one obtains

$$
\Phi_{i, k}(\overrightarrow{\mathrm{q}})=C_{i s, k s^{\prime}} q_{s} q_{s^{\prime}} \cdot
$$

Accordingly $\Phi_{i, k}(\overrightarrow{\mathrm{q}})$ is identical with the inverse propagator of the usual continuum theory as one 
expects. Introducing the real-space Green's function via

$$
G_{i, k}\left(\overrightarrow{\mathrm{r}}-\overrightarrow{\mathrm{r}}^{\sigma}\right)=\frac{1}{(2 \pi)^{3}} \int d^{3} q \Phi_{i, k}^{-1}(\overrightarrow{\mathrm{q}}) e^{i \overrightarrow{\mathrm{q}} \cdot\left(\overrightarrow{\mathrm{r}}-\overrightarrow{\mathrm{r}}^{\sigma}\right)},
$$

the Fourier transform of Eq. (26) can be written in the form

$$
\begin{array}{r}
p_{j}(\overrightarrow{\mathrm{r}})=-\frac{1}{2} \sum_{\sigma} \int_{\Sigma_{\sigma}} d^{2} r_{s}^{\sigma}\left(C_{i s, k s^{\prime}}+C_{i s^{\prime}, k s}\right) \\
\cdot \quad \times b_{i}^{\sigma} \frac{\partial}{\partial r_{s^{\prime}}} G_{j, k}\left(\overrightarrow{\mathrm{r}}-\overrightarrow{\mathrm{r}}^{\sigma}\right) .
\end{array}
$$

Accordingly Eq. (29) goes over into the standard displacement field produced by dislocation ${ }^{4}$ only if

$$
C_{i s, k s^{\prime}}=C_{i s^{\prime}, k s}
$$

holds. Together with the other symmetry conditions which $C_{i j, n m}$ satisfies this implies that Hook's tensor has to be symmetrical in all its indices. Accordingly the present defect model goes over into the usual crystalline model only for lattice constituents interacting via central forces. It is obvious that if the displacement fields coincide that also the interaction energies of both theories have to be same. This follows from the fact that the energy functionals $\Phi$ of both theories must coincide in those terms which are of first and second order in the variables $\{\overrightarrow{\mathrm{S}} \overrightarrow{\mathrm{H}}\}$ in order that the displacement fields $\left\{\vec{p}^{\vec{t}}\right\}$ coincide, and this is the case. There remains therefore only the possibility that the two energy functionals differ in a term which is bilinear in the fields $\{\overrightarrow{\mathrm{b}} \overrightarrow{\mathrm{n}}, \overrightarrow{\mathrm{m}}\}$. Such a term can only involve couplings over the coupling-constant matrix $\left\{\Phi_{i, j}^{\overrightarrow{\mathrm{n}}, \overrightarrow{\mathrm{m}}}\right\}$ but no long-range couplings which are only mediated over the phonon propagator and cannot appear in a microscopic Hamiltonian and also not in a phenomenological theory. Accordingly the interaction law between defects which is of long range has to coincide in both theories. There may, however, be a difference in the core energy.

In order to simplify Eq. (26) further it will now be transformed onto the orthogonal trihedron $\left(\overrightarrow{\mathrm{n}}_{1^{\prime}}(\overrightarrow{\mathrm{q}}), \overrightarrow{\mathrm{n}}_{2^{\prime}}(\overrightarrow{\mathrm{q}}), \overrightarrow{\mathrm{n}}_{3^{\prime}}(\overrightarrow{\mathrm{q}})\right)$, with $\overrightarrow{\mathrm{n}}_{3^{\prime}}(\overrightarrow{\mathrm{q}})=\overrightarrow{\mathrm{q}}^{0}$. Because Eq. (26) is written in a covariant way it preserves its form when refered to a primed coordinate system, i.e.,

$$
\begin{aligned}
p_{-\overrightarrow{\mathrm{q}}, j^{\prime}}=-\frac{1}{\sqrt{N}} \Phi_{j^{\prime}, k^{\prime}}^{-1}(\overrightarrow{\mathrm{q}}) \frac{1}{2} \sum_{\sigma, \overrightarrow{\mathrm{h}}^{\prime}} \int_{\Sigma_{\sigma}}\left(d^{2} \overrightarrow{\mathrm{r}}^{\sigma} \cdot \overrightarrow{\mathrm{h}}\right) \Phi_{k^{\prime}, i^{\prime}}^{\overrightarrow{\mathrm{h}}} \\
\times b_{i^{\prime}}^{\sigma}(\overrightarrow{i \mathrm{q}} \cdot \overrightarrow{\mathrm{h}}) e^{-i \overrightarrow{\mathrm{q}} \cdot \overrightarrow{\mathrm{r}}^{\sigma}} .
\end{aligned}
$$

Using Eq. (9) in the primed coordinate system and which consists of the trihedron introduced above and observing $(\overrightarrow{\mathrm{q}} \cdot \overrightarrow{\mathrm{h}})=q h_{3^{\prime}}$ allows one to write Eq. (31) in the form

$$
\begin{aligned}
p_{-\vec{q}, j^{\prime}}=\frac{1}{\sqrt{N}} \Phi_{j^{\prime}, k^{\prime}}^{-1}(\overrightarrow{\mathrm{q}}) \sum_{\sigma} & i q \int_{\Sigma_{\sigma}} d^{2} r^{\sigma} n_{s^{\prime}}\left(\overrightarrow{\mathrm{r}}^{\sigma}\right) \widetilde{C}_{k^{\prime} i^{\prime}, s^{\prime} 3^{\prime}} \\
& \times b_{i^{\prime}}^{\sigma}, e^{-i \overrightarrow{\mathrm{q}} \circ \overrightarrow{\mathrm{I}}^{\sigma} \sigma}
\end{aligned}
$$

where

$$
\overrightarrow{\mathrm{n}}\left(\overrightarrow{\mathrm{r}}^{\sigma}\right)=\left(n_{1^{\prime}}\left(\overrightarrow{\mathrm{r}}^{\sigma}\right), n_{z^{\prime}}\left(\overrightarrow{\mathrm{r}}^{\sigma}\right), n_{3^{\prime}}\left(\overrightarrow{\mathrm{r}}^{\sigma}\right)\right)
$$

represents the normal vector of the cut surface. This normal vector can be put into the form

$$
n_{s^{\prime}}\left(\overrightarrow{\mathrm{r}}^{\sigma}\right)=I_{s^{\prime} k^{\prime}}\left(q^{0} \times \overrightarrow{\mathrm{n}}\left(\overrightarrow{\mathrm{r}}^{\sigma}\right)\right)_{k^{\prime}}+n_{3^{\prime}}\left(\overrightarrow{\mathrm{r}}^{\sigma}\right) \delta_{s^{\prime}, 3^{\prime}},
$$

where

$$
I=\left(\begin{array}{ccc}
0 & 1 & 0 \\
-1 & 0 & 0 \\
0 & 0 & 0
\end{array}\right)
$$

has been introduced. Inserting Eq. (33) into Eq. (32) yields

$p_{-\overrightarrow{\mathrm{q}}, j^{\prime}}=\frac{1}{\sqrt{N}} \Phi_{j^{\prime} k^{\prime}}^{-1}(\overrightarrow{\mathrm{q}}) i q \sum_{\sigma} \int_{\Sigma_{\sigma}} d^{2} r^{\sigma} n_{3^{\prime}}\left(\overrightarrow{\mathrm{r}}^{\sigma}\right) \tilde{C}_{k^{\prime} i^{\prime}, 3^{\prime} 3^{\prime}}, b_{i^{\prime}}^{\sigma} e^{-i \mathrm{q} \cdot \overrightarrow{\mathrm{r}}^{\sigma}}+\frac{1}{\sqrt{N}} \Phi_{j^{\prime}, k^{\prime}}^{-1}(\overrightarrow{\mathrm{q}}) \sum_{\sigma} \int_{\Sigma_{\sigma}} I_{s^{\prime} \boldsymbol{p}^{\prime}}\left(i \overrightarrow{\mathrm{q}} \times d^{2} \overrightarrow{\mathrm{r}}^{\sigma}\right)_{p^{\prime}} \tilde{C}_{k^{\prime} i^{\prime}, s^{\prime} 3^{\prime}}, b_{i^{\prime}}^{\sigma} e^{-i \mathrm{q} \cdot \overrightarrow{\mathrm{r}}^{\sigma}}$.

Using Stokes' theorem in the form of Eq. (A17) of the Appendix Eq. (34) can be transformed into

$p_{-\overrightarrow{\mathrm{q}}, \boldsymbol{j}^{\prime}}=\frac{1}{\sqrt{N}} \Phi_{j^{\prime}, k^{\prime}}^{-1}(\overrightarrow{\mathrm{q}}) i \sum_{\sigma} \int_{\Sigma_{\sigma}}\left(d^{2} \overrightarrow{\mathrm{r}}^{\sigma}\right)_{3^{\prime}} q \tilde{C}_{k^{\prime} i^{\prime}, 3^{\prime} 3^{\prime}}, b_{i^{\prime}}^{\sigma}, e^{-\overrightarrow{\mathrm{q}} \cdot \overrightarrow{\mathrm{r}}^{\sigma}}+\frac{1}{\sqrt{N}} \Phi_{j^{\prime}, k^{\prime}}^{-1}(\overrightarrow{\mathrm{q}}) \sum_{\sigma} \int_{C_{\sigma}}\left(d \overrightarrow{\mathrm{r}}^{\sigma} \times \overrightarrow{\mathrm{q}}^{0}\right)_{s^{\prime}} \tilde{C}_{k^{\prime} i^{\prime}, s^{\prime}, 3^{\prime}}, b_{i^{\prime}}^{\sigma}, e^{-i \overrightarrow{\mathrm{q}} \cdot \overrightarrow{\mathbf{r}}^{\sigma}}$.

Observing that

$$
\tilde{C}_{i^{\prime} k^{\prime}, 3^{\prime} 3^{\prime}}=\tilde{C}_{i^{\prime} k^{\prime}, n m} q_{n}^{0} q_{m}^{0}, \quad C_{i^{\prime} k^{\prime}, s^{\prime} 3^{\prime}}=C_{i^{\prime} k^{\prime}, s^{\prime} n} q_{n}^{0}
$$

holds, Eq. (35) can be expressed in the original coordinate system. This yields

$$
p_{-\overrightarrow{\mathrm{q}}, j}=\frac{1}{\sqrt{N}} \Phi_{j, k}^{-1}(\overrightarrow{\mathrm{q}}) i \sum_{\sigma} \int_{\Sigma_{\sigma}}\left(d^{2} \overrightarrow{\mathrm{r}}^{\sigma} \cdot \overrightarrow{\mathrm{q}}\right) \tilde{C}_{k i, n m} b_{i}^{\sigma} q_{n}^{0} q_{m}^{0} e^{-i \mathrm{q} \cdot \overrightarrow{\mathrm{r}}^{\sigma}}+\frac{1}{\sqrt{N}} \Phi_{j, k}^{-1}(\overrightarrow{\mathrm{q}}) \sum_{\sigma} \int_{C_{\sigma}}\left(d \overrightarrow{\mathrm{r}}^{\sigma} \times \overrightarrow{\mathrm{q}}^{0}\right)_{s} \tilde{C}_{k i, s n} q_{n}^{0} b_{i}^{\sigma} e^{-i \mathrm{q} \cdot \overrightarrow{\mathrm{r}}^{\sigma}} .
$$


A different derivation of this formula using pure vector algebra leads naturally to the same result. The first term of Eq. (36) involves surface integrals which lead to discontinuities of $\vec{p}(\vec{r})$ over the cut surfaces, whereas the second part of Eq. (36) represents the physically relevant displacement field which is continuous.

It has been shown in this section that only for central forces where Eq. (30) holds the microscopic defect model defined by Eqs. (7) and (8) leads to the standard dislocation theory based on phenomenological elasticity theory. Suppose now that standard dislocation theory satisfies Eq. (18) (which it necessarily must do if it is correct) just by way of its construction. In the case of noncentral forces Eq. (7) has then to be modified in order to exhibit rotational invariance. There are a number of possibilities to do that, but so far we have found no systematic way to do it successfully. Because noncentral forces are usually generated by couplings to the electron system it may be necessary that in that case Eq. (7) has to be replaced by an electron lattice Hamiltonian. Elimination of the degrees of freedom of the electron system might then lead to the correct invariance properties of the renormalized lattice Hamiltonian. This has not been done so far.

\section{ISOTROPIC MEDIUM}

In this section we evaluate the interaction energy and displacement field in the presence of dislocations in the isotropic case.

It has been pointed out by Huang ${ }^{9}$ that in an infinite lattice two independent conditions have to be fulfilled by the coupling-constant matrices besides the conditions imposed by symmetry in order that a stable elastic medium results. First, linear terms in the Taylor expansion of the interaction energy have to vanish in order that each lattice point is in equilibrium. Second, all stresses have to vanish which imposes an independent (not) symmetry (implied) condition on the tensor $\tilde{C}_{i k, n m}$ defined in Eq. (9), namely [see Eq. (58.7) of Ref. 8],

$$
\tilde{C}_{i k, m n}=\tilde{C}_{m n, i k} .
$$

This condition is, however, always satisfied in cubic lattices [see Eq. (37.10) of Ref. 8] once the coupling-constant matrices satisfy the symmetry conditions. For isotropic media one gets according to Leibfried ${ }^{8}$

$$
\tilde{C}_{i k, m n}=\tilde{C}_{12} \delta_{i k} \delta_{m n}+\tilde{C}_{44}\left(\delta_{i m} \delta_{k n}+\delta_{i n} \delta_{k m}\right)
$$

and

$$
\tilde{C}_{11}=\tilde{C}_{12}+2 \tilde{C}_{44} \text {. }
$$

The constants appearing in Eqs. (38) and (39) are related to Voigt's elastic constants in a cubic medium by the equations

$$
C_{11}=\tilde{C}_{11}, \quad C_{12}=2 \tilde{C}_{44}-\tilde{C}_{12}, \quad \tilde{C}_{44}=\tilde{C}_{12} .
$$

It is clear that the stability criteria mentioned above imply only that the phonon spectrum of our Hamiltonian Eq. (7) is positive definite but not that Eq. (7) is stable against defect generation. A more detailed discussion of the stability properties of Eq. (7) will be postponed to the end of this section.

Next we evaluate the interaction energy $\Phi^{\text {int }}$ and displacement $\vec{p}(\vec{r})$ for the isotropic case. Abbreviating the inverse propagator Eq. $\left(27^{\prime}\right)$ in the form

$$
\Phi_{i, k}(\vec{q})=q^{2}\left(A \delta_{i k}+B q_{i}^{0} q_{k}^{0}\right),
$$

one obtains

$$
\Phi_{i, k}^{-1}=\left(1 / q^{2}\right)\left(C \delta_{i k}+D q_{i}^{0} q_{k}^{0}\right)
$$

where

$$
\begin{array}{ll}
A \equiv \tilde{C}_{12}, & B \equiv 2 \tilde{C}_{44}, \\
C \equiv 1 / A, & D \equiv-(B / A) /(A+B)
\end{array}
$$

has been introduced. It is now straightforward but tedious to evaluate Eq. (24). One obtains

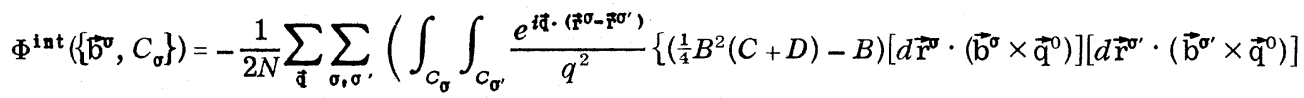

$$
\begin{aligned}
& \left.\left.+\left(d r^{\sigma} \times \overrightarrow{\mathrm{q}}^{0}\right) \cdot\left(d \overrightarrow{\mathrm{r}}^{\boldsymbol{\beta}^{\prime}} \times \overrightarrow{\mathrm{q}}^{0}\right)\left[\frac{1}{4} B^{2} C\left(\overrightarrow{\mathrm{b}}^{\sigma} \cdot \overrightarrow{\mathrm{q}}^{0}\right)\left(\overrightarrow{\mathrm{b}}^{\sigma^{\prime}} \cdot \overrightarrow{\mathrm{q}}^{0}\right)-A\left(\overrightarrow{\mathrm{b}}^{\sigma} \cdot \overrightarrow{\mathrm{b}}^{\sigma^{\prime}}\right)\right]\right\}\right) .
\end{aligned}
$$

Evaluating the coefficients in Eq. (43) and going over to a real-space representation yields

$$
\begin{aligned}
& \Phi^{\text {int }}\left(\left\{\overrightarrow{\mathrm{b}}^{\sigma}, C_{\sigma}\right\}\right)=-\frac{1}{2} \sum_{\sigma_{\sigma} \sigma^{\prime}}\left(\frac{\mu}{4(1-\nu)} \frac{7-8 \nu}{2(1-2 \nu)} \frac{1}{8 \pi} \int_{C_{\sigma}} \int_{C_{\sigma^{\prime}}}\left[d \overrightarrow{\mathrm{r}}^{\sigma} \cdot\left(\overrightarrow{\mathrm{b}}^{\sigma} \times \vec{\nabla}_{\overrightarrow{\mathrm{r}}^{\sigma}}\right)\right]\left[d \overrightarrow{\mathrm{r}}^{\sigma^{\prime}} \cdot\left(\overrightarrow{\mathrm{b}}^{\sigma^{\prime}} \times \vec{\nabla}_{\overrightarrow{\mathrm{r}}^{\prime} \sigma^{\prime}}\right)\right]\left|\overrightarrow{\mathrm{r}}^{\sigma}-\overrightarrow{\mathrm{r}}^{\sigma^{\prime}}\right|\right. \\
& +\frac{\mu}{4} \frac{1}{(1-2 \nu)^{2}} \frac{1}{12 \times 8 \pi} \int_{C_{\sigma}} \int_{C_{\sigma^{\prime}}}\left(d \overrightarrow{\mathrm{r}}^{\sigma} \times \vec{\nabla}_{\overrightarrow{\mathbf{F}^{\sigma}}}\right) \cdot\left(d \overrightarrow{\mathrm{r}}^{\sigma^{\prime}} \times \vec{\nabla}_{\overrightarrow{\mathrm{r}}^{\prime}}\right)\left(\overrightarrow{\mathrm{b}}^{\sigma} \cdot \vec{\nabla}_{\mathbf{F}^{\sigma}}\right)\left(\overrightarrow{\mathrm{b}}^{\sigma^{\prime}} \cdot \vec{\nabla}_{\overrightarrow{\mathbf{r}}^{\sigma^{\prime}}}\right)\left|\overrightarrow{\mathrm{r}}^{\sigma}-\overrightarrow{\mathrm{r}}^{\sigma^{\prime}}\right|^{3} \\
& \left.+\mu \frac{1}{8 \pi}\left(\overrightarrow{\mathrm{b}}^{\sigma} \cdot \overrightarrow{\mathrm{b}}^{\sigma^{\prime}}\right) \int_{C_{\sigma}} \int_{C_{\sigma^{\prime}}}\left(d \overrightarrow{\mathrm{r}}^{\sigma} \times \vec{\nabla}_{\overrightarrow{\mathbf{r}}^{\sigma}}\right) \cdot\left(d \overrightarrow{\mathrm{r}}^{\sigma^{\prime}} \times \vec{\nabla}_{\overrightarrow{\mathbf{r}}^{\prime}}\right)\left|\overrightarrow{\mathrm{r}}^{\sigma}-\overrightarrow{\mathrm{r}}^{\sigma^{\prime}}\right|\right) .
\end{aligned}
$$


Similarly one obtains for Eq. (36)

$$
\begin{aligned}
\overrightarrow{\mathrm{p}}_{-\overrightarrow{\mathrm{q}}}=\frac{1}{q^{2}} \frac{1}{\sqrt{N}} \sum_{\sigma} A C \overrightarrow{\mathrm{b}}^{\sigma} i \int_{\Sigma_{\sigma}}\left(d^{2} \overrightarrow{\mathrm{r}}^{\sigma} \cdot \overrightarrow{\mathrm{q}}\right) e^{-i \overrightarrow{\mathrm{q}} \cdot \overrightarrow{\mathrm{r}}^{\sigma}}+\frac{1}{q^{2}} \frac{B}{2} \frac{1}{\sqrt{N}} \sum_{\sigma} & \left(C \int_{C_{\sigma}}\left(d \overrightarrow{\mathrm{r}}^{0} \times \overrightarrow{\mathrm{q}}^{0}\right)\left(\overrightarrow{\mathrm{q}}^{0} \cdot \overrightarrow{\mathrm{b}}^{\sigma}\right) e^{-i \mathrm{q} \cdot \overrightarrow{\mathrm{r}}^{\sigma}}\right. \\
& +(C+D) \int_{C_{\sigma}}\left(d \overrightarrow{\mathrm{r}}^{\sigma} \times \overrightarrow{\mathrm{q}}^{0}\right) \cdot \overrightarrow{\mathrm{b}}^{\sigma} \overrightarrow{\mathrm{q}}^{0} e^{\left.-i \mathrm{q} \cdot \overrightarrow{\mathrm{r}}^{\sigma}\right)} .
\end{aligned}
$$

In real-space representation one obtains from Eq. (44) using $B C=1 /(1-2 \nu)$ and $B(C+D)=1 /[2(1-\nu)]$ and

$$
\frac{1}{8 \pi^{3}} \int_{\epsilon}^{\infty} \frac{d^{3} q}{q^{4}} e^{i \vec{q} \cdot \overrightarrow{\mathbf{r}}}=-\frac{r}{8 \pi}+\frac{1}{2 \pi^{2} \epsilon}
$$

where $\epsilon \ll 1$ is a cutoff in $\overrightarrow{\mathrm{q}}$ space

$$
\begin{aligned}
\overrightarrow{\mathrm{p}}(\overrightarrow{\mathrm{r}})=\frac{1}{4 \pi} \sum_{\sigma} \overrightarrow{\mathrm{b}}^{\sigma} \Omega^{\sigma}(\overrightarrow{\mathrm{r}})+\frac{1}{16 \pi} \sum_{\sigma} & {\left[\frac{1}{1-2 \nu}\left(\int_{C_{\sigma}} \frac{\left(d \overrightarrow{\mathrm{r}}^{\sigma} \times \overrightarrow{\mathrm{b}}^{\sigma}\right)}{\left|\overrightarrow{\mathrm{r}}^{\sigma}-\overrightarrow{\mathrm{r}}\right|}-\int_{C_{\sigma}} \frac{d \overrightarrow{\mathrm{r}}^{\sigma} \times\left(\overrightarrow{\mathrm{r}}^{\sigma}-\overrightarrow{\mathrm{r}}\right)}{\left|\overrightarrow{\mathrm{r}}^{\sigma}-\overrightarrow{\mathrm{r}}\right|^{2}} \frac{\left(\overrightarrow{\mathrm{r}}^{\sigma}-\overrightarrow{\mathrm{r}}\right) \cdot \overrightarrow{\mathrm{b}}^{\sigma}}{\left|\overrightarrow{\mathrm{r}}^{\sigma}-\overrightarrow{\mathrm{r}}\right|}\right)\right.} \\
& \left.-\frac{1}{2(1-\nu)} \vec{\nabla}_{\overrightarrow{\mathrm{r}}} \int_{C_{\sigma}} \frac{\left[d \overrightarrow{\mathrm{r}}^{\sigma} \times\left(\overrightarrow{\mathrm{r}}^{\sigma}-\overrightarrow{\mathrm{r}}\right)\right] \cdot \overrightarrow{\mathrm{b}}^{\sigma}}{\left|\overrightarrow{\mathrm{r}}^{\sigma}-\overrightarrow{\mathrm{r}}\right|}\right],
\end{aligned}
$$

where as usual the space angle

$$
\Omega^{\sigma}(\overrightarrow{\mathrm{r}}) \equiv \int_{\Sigma_{\sigma}} \frac{d^{2} \overrightarrow{\mathrm{r}}^{\sigma} \cdot\left(\overrightarrow{\mathrm{r}}^{\mathrm{\sigma}}-\overrightarrow{\mathrm{r}}\right)}{\left|\overrightarrow{\mathrm{r}}^{\sigma}-\overrightarrow{\mathrm{r}}\right|^{2}}
$$

has been introduced.

Comparison of $\Phi^{\mathbf{i n t}}\left(\left\{\overrightarrow{\mathrm{b}}^{\boldsymbol{\sigma}}, C_{\boldsymbol{\sigma}}\right\}\right)$ and $\overrightarrow{\mathrm{p}}(\overrightarrow{\mathrm{r}})$ with the corresponding expressions given, e.g., in Ref. 3 under Eq. (2.28) reveals that the interaction laws between the "dislocations" defined in our model and the displacements produced by them do not agree with the standard expressions obtained by means of the phenomenological dislocation theory. Although in both cases interaction between various parts of the dislocation loops is mediated by a direction-dependent Coulomb law, the re are two main differences. First, the Poisson coefficient enters in a different way leading to a divergence of $\Phi^{\text {int }}$ for $\nu \rightarrow \frac{1}{2}$ and $\mu \neq 0$. Second the direction dependence of the interaction law is different. With respect to the first point we have emphasized at the beginning of Sec. II that the save stability range of the present model Hamiltonian is $C_{12}>0$ and $C_{44}>0$, where in addition Eq. (48) has to be satisfied. This implies as will be shown below that $\nu \leqslant \frac{1}{4}$ has to hold. Accordingly instability of the model Hamiltonian Eq. (7) when used in the interval $\frac{1}{4} \leqslant \nu \leqslant \frac{1}{2}$ is possible. Concerning the difference in direction dependence of the interaction law the reason is that Eq. (7) only for the case of central-force interaction exhibits all the proper symmetry and stability conditions required in order that it describes defects of dislocation type as occuring in solids. For the isotropic model this implies $\nu=\frac{1}{4}$ and $\mu$ arbitrary as will be shown presently.

In the presence of dislocations internal stresses in the medium are generated and we have pointed out in Sec. II that in order that the medium is stable against the production of defects a sufficient condition is that all $3 \times 3$ matrices $\left\{-\Phi_{i, k}^{h \neq 0}\right\}_{h}$ shall be positive definite, whereas positive definiteness of the phonon spectrum requires $C_{12}>0$ and $C_{44}>0$. Although the $3 \times 3$ matrices $\left\{-\Phi_{\substack{\overrightarrow{\mathrm{h}} \neq 0 \\ i, k}}\right\}_{\mathrm{h}}$, can always be made positive definite by means of their diagonal elements and this implies via Eq. (20) that also the phonon spectrum can be made positive definite it is not clear if the extra condition Eq. (38) can be fulfilled simultaneously. The point here is that the isotropic case of a cubic lattice as defined by Eqs. (38) and (39) is much more restrictive for the present theory than for the anisotropic cubic lattice. For the latter case the potential described by Eq. (7) may have the required stability properties only for certain parts of the coupling-constant space. The requirement of positive definiteness on each matrix $\left\{-\Phi_{i, k}^{\hat{h}}\right\}_{\mathbf{h}}$ in the presence of $n$ th-order neighbor coupling is, of course, rather strong. The reason for this is that over the cut surface many lattice sites are coupled and that therefore only the tensor $\tilde{C}_{i k, m n}$ determines the stability of the system against the generation of topological objects. This holds at least in the long-wavelength approximation used in this paper. Checking, however, the stability of the system only via the $\tilde{C}_{i k, m n}$ tensor or equivalently the Voigt elastic constants $\left\{C_{i j}\right\}$ requires the analysis of the interaction energy of all defect configurations in order to confirm that $\Phi^{\text {int }}>0$ holds true always. In the following a simpler method will be applied by considering a model which involves only first-, second- and third-order neighbors.

According to Table 9 of Ref. 8 the couplingconstant matrices for that case can be represented in the form 


$$
\begin{aligned}
& \left\{\Phi_{i, k}^{\overrightarrow{\mathrm{h}=}=(100)}\right\}=\left[\begin{array}{lll}
\alpha & 0 & 0 \\
0 & \beta & 0 \\
0 & 0 & \beta
\end{array}\right), \\
& \left\{\Phi_{i, k}^{\overrightarrow{\mathrm{h}}=(110)}\right\}=\left[\begin{array}{lll}
\beta^{\prime} & \gamma^{\prime} & 0 \\
\gamma^{\prime} & \beta^{\prime} & 0 \\
0 & 0 & \alpha^{\prime}
\end{array}\right), \\
& \left\{\Phi_{i, k}^{\overrightarrow{\mathrm{h}}=(111)}\right\}^{\prime}=\left(\begin{array}{lll}
\alpha^{\prime \prime} & \gamma^{\prime \prime} & \gamma^{\prime \prime} \\
\gamma^{\prime \prime} & \alpha^{\prime \prime} & \gamma^{\prime \prime} \\
\gamma^{\prime \prime} & \gamma^{\prime \prime} & \alpha^{\prime \prime}
\end{array}\right)
\end{aligned}
$$

and all others can be obtained by means of symmetry operations $D^{s}$. In a cubic medium the seven coupling constants appearing in Eq. (45) determine via three equations the Voigt elastic constants and no further condition is imposed by Eq. (37) on the range of variability of the coupling constants. For the isotropic case of a cubic lattice one uses Eqs. (27), (38), (39), (40), and (45) to obtain

$$
\begin{aligned}
& C_{12}=-4 \gamma^{\prime}-8 \gamma^{\prime \prime}+\left(\beta+2 \alpha^{\prime}+2 \beta^{\prime}+4 \alpha^{\prime \prime}\right), \\
& C_{44}=-\left(\beta+2 \alpha^{\prime}+2 \beta^{\prime}+4 \alpha^{\prime \prime}\right), \\
& \alpha=\beta+2 \alpha^{\prime}-2 \beta^{\prime}+4 \gamma^{\prime}+8 \gamma^{\prime \prime} .
\end{aligned}
$$

Here Eq. (46c) implies the isotropy of the medium. In order that the $3 \times 3$ matrices $\left\{-\Phi_{i k}^{\overrightarrow{\mathbf{h}}}\right\}_{h}$ are positive definite

$$
\begin{aligned}
& \alpha<0, \quad \beta<0, \quad \alpha^{\prime}<0, \quad \beta^{\prime}<0, \quad\left|\gamma^{\prime}\right|<\left|\beta^{\prime}\right|, \\
& \alpha^{\prime \prime}<0, \quad \alpha^{\prime \prime}<\gamma^{\prime \prime}<-\frac{1}{2} \alpha^{\prime \prime}
\end{aligned}
$$

is required. This implies that $C_{44}>0$ is satisfied automatically. Furthermore

$$
-4 \gamma^{\prime}-8 \gamma^{\prime \prime}+2 \beta^{\prime}>\left|\beta+2 \alpha^{\prime}+4 \alpha^{\prime \prime}\right|
$$

is required for $C_{12}>0$ implying together with Eq. $(47 \mathrm{~b}), \gamma^{\prime}<0$. Equations (47a) and (47c) imply that Eq. (46c) can also be satisfied. It follows from this that for the present model Eq. (7) represents a stable potential except for the pathological cases like

$$
\beta=\alpha^{\prime}=\beta^{\prime}=\alpha^{\prime \prime}=0
$$

which implies via Eqs. (47a) and (47b)

$$
\gamma^{\prime}=\gamma^{\prime \prime}=0
$$

and the refore $C_{12}=C_{44}=0$. The maximal value of $C_{12}$ for given $C_{44}$ is realized for $\gamma^{\prime}=\beta^{\prime}$, and $\gamma^{\prime \prime}$

$=\alpha^{\prime \prime}$. Inserting these values into Eq. (46a) yields

$$
\max \left(C_{12}\right)=C_{44}+2 \beta+4 \alpha^{\prime}
$$

and consequently

$$
C_{12} \leqslant C_{44} \text {. }
$$

If one introduces the shear modulus $\mu$ and the Poisson coefficient $\nu$ then

$$
C_{12}=2 \mu \nu /(1-2 \nu), \quad C_{44}=\mu
$$

holds and Eq. (48) reads $\nu \lesssim \frac{1}{4}$. Within the present f ramework, i.e., taking coupling constants up to third-order neighbors into account and imposing the strong stability condition of positive definiteness on all three $\left\{-\Phi_{i, k}^{\vec{h}}\right\}_{h}$-coupling-constant matrices the limiting case $\mu=0$ but $C_{12} \neq 0$ can not be approached properly. It follows from the present analysis that at least for the range of coupling constants indicated by Eqs. (47a)-(47c) the potential Eq. (7) is stable with respect to phonon excitations and defect generation.

\section{DISCUSSION AND CONCLUSION}

In the present paper we have tried to develop a defect theory for a simple-cubic crystal. The method has been based on an ad hoc ansatz for the microscopic crystal potential. This potential is referred to a specific symmetry-broken state of the system and its metastable states can be associated with "dislocation" loops. These dislocations are characterized by a Burgers vector which obeys the same conservation laws as those obeyed by the usual dislocations. The dislocations interact via a Biot-Savard like inte raction. Furthermore all interaction can be expressed in the long-wavelength approximation in terms of the macroscopic elastic constants. Despite that the present dislocations differ from the usual ones because the elastic constants and the directional dependence of the interaction law enters in a different way in the presence of noncentral twoparticle interaction. The origin of that disagreement originates from the lack of rotational invariance of the Hamiltonian Eq. (7) for noncentral interaction. Although the propagator and therefore the phonon dispersions in the dislocated state have the correct symmetry a uniform rotation of the crystal does not drag along the defects in a proper way in the general case. Unfortunately we have not been able to get rid of that deficiency. It is proposed, however, either to add symmetry restoring terms to Eq. (7) or to refer the crystal Hamiltonian to a local coordinate system defined by the displacements $\left\{\vec{p}\left(\vec{R}^{\mathbb{z}}\right) *\right\}$ [obtained from Eq. (36) modulo the first term of this expression] which originate from the lattice defects. The problem of noncentral forces can be treated in a formal way as follows. Leibfried in Ref. 8 (see footnote 1 , Sect. 58) points out that only the lattice energy density

$$
\Phi(\overrightarrow{\mathrm{r}})=\frac{1}{2} \sum_{\substack{i, m \\ k, n}}\left[\tilde{C}_{i_{k, m n}}+\tilde{C}_{m k, i_{n}}-\tilde{C}_{m i, n k}\right] S_{i, m}(\overrightarrow{\mathrm{r}}) S_{k, n}(\overrightarrow{\mathrm{r}})
$$


has the correct rotational symmetry. For displacement fields vanishing at infinity the second and third terms of Eq. (50), which is a divergence vanish, however, and Eq. (50) is the continuum limit of the density corresponding to Eq. (1). In the presence of dislocations the second and third terms in Eq. (50) do not vanish. The lattice potential corresponding to Eq. (50) may be written in the form

$$
\begin{aligned}
\Phi(\{\overrightarrow{\mathrm{R}} \mathrm{\textrm {n }}\})= & -\frac{1}{4} \sum_{\substack{\overrightarrow{\mathrm{m}}, \overrightarrow{\mathrm{n}} \\
i, k}} \Phi_{i, k}^{\overrightarrow{\mathrm{m}}, \overrightarrow{\mathrm{n}}} S_{i}^{\overrightarrow{\mathrm{m}}-\overrightarrow{\mathrm{n}}} S_{k}^{\overrightarrow{\mathrm{m}}-\overrightarrow{\mathrm{n}}} \\
& -\frac{1}{4} \sum_{\substack{\mathrm{m}, \overrightarrow{\mathrm{n}} \\
i, k}}\left\{\Phi_{s, k}^{\overrightarrow{\mathrm{m}}, \overrightarrow{\mathrm{n}}} \frac{h_{i}}{h_{s}}-\Phi_{s, i}^{\overrightarrow{\mathrm{m}}, \overrightarrow{\mathrm{n}}} \frac{h_{k}}{h_{s}}\right\} S_{i}^{\overrightarrow{\mathrm{m}}-\overrightarrow{\mathrm{n}}} S_{k}^{\overrightarrow{\mathrm{m}}-\overrightarrow{\mathrm{n}}},
\end{aligned}
$$

where $\overrightarrow{\mathrm{h}}=R_{0}^{\overrightarrow{\mathrm{m}}}-R_{0}^{\overrightarrow{\mathrm{n}}}$. Next one makes the replacement Eq. (6). Then the second term of Eq. (51) gives the non-central-force contribution to the dislocation theory. The displacement field $\overrightarrow{\mathrm{p}}^{n}(\overrightarrow{\mathrm{r}})$ due to this contribution is easily calculated using the methods outlined in the Appendix. One obtains

$$
\begin{gathered}
p_{j}^{n c}(\overrightarrow{\mathrm{r}})=\frac{1}{2} \sum_{\sigma} \sum_{\overrightarrow{\mathrm{h}}} \int_{\Sigma_{\sigma}}\left(d^{2} \overrightarrow{\mathrm{r}}^{\sigma} \cdot \overrightarrow{\mathrm{h}}\right)\left[\Phi_{s, i}^{\overrightarrow{\mathrm{h}}} b_{i}^{\sigma} h_{k}-\Phi_{s^{\prime}, k}^{\overrightarrow{\mathrm{h}}}\left(\overrightarrow{\mathrm{h}} \cdot \overrightarrow{\mathrm{b}}^{\sigma}\right)\right] \\
\times \frac{\partial}{\partial r_{s^{\prime}}} G_{j k}\left(\overrightarrow{\mathrm{r}}-\overrightarrow{\mathrm{r}}^{\sigma}\right) .
\end{gathered}
$$

Using Eq. (9) and [see Ref. 8, Eq. (58.9)]

$$
C_{k s^{\prime}, i s}=\tilde{C}_{k i, s s^{\prime}}+\tilde{C}_{s^{\prime} i, k s}-\tilde{C}_{s^{\prime} k, s i}
$$

and adding Eq. (52) to Eq. (29) where Eq. (28) is observed leads to the standard displacement field produced by dislocations for non-central-force interactions. The evaluation of the interaction energy is more cumbersome and so far has not been done.

\section{ACKNOWLEDGMENTS}

In conclusion the author kindly acknowledges stimulating discussion with Professor, P. F. Choquard, Professor H. Gleiter, Professor E. Kröner, and Professor G. Meissner on the problem of defect states in solids. The author's stay at the Instituto de Fisica da Universidade Federal do Rio Grande do Sul has been arranged within the agreement DAAD (Germany)-CNP (Brazil). The kind hospitality at the Instituto de Fisica is acknowledged.

\section{APPENDIX: DERIVATION OF THE INTERACTION LAW}

In order to simplify Eq. (23) we first study the term

$$
I_{j} \equiv \sum_{\overrightarrow{\mathrm{m}}, \overrightarrow{\mathrm{n}}} \Phi_{\vec{j}, s}^{\overrightarrow{\mathrm{m}}-\overrightarrow{\mathrm{n}}} b_{s}^{\overrightarrow{\mathrm{m}}-\overrightarrow{\mathrm{n}}} e^{i \overrightarrow{\mathrm{i}} \overrightarrow{\mathrm{e}} \cdot \overrightarrow{\mathrm{R}} \overrightarrow{\mathrm{R}}_{0}^{\overrightarrow{\mathrm{m}}}}
$$

Assume first that there is one cut surface $\Sigma_{\sigma}$ as illustrated in Fig. 1 and that the two sides of the cut surface are displaced by a vector $\vec{b}^{\sigma}$. It then follows that the displacement vectors $\overrightarrow{\vec{b}^{\mathrm{m}}}$, $\vec{i}$ for $\vec{R}_{0}^{\vec{m}}$ and $\vec{R}_{0}^{\mathrm{n}}$ pointing versus opposite sides of the cut surface fulfill

$$
\overrightarrow{\mathrm{b}}^{\overrightarrow{\mathrm{m}}, \overrightarrow{\mathrm{n}}}=\overrightarrow{\mathrm{b}}^{\sigma}
$$

and otherwise

$$
\overrightarrow{\mathrm{b}^{\mathrm{m}}, \overrightarrow{\mathrm{i}}}=0 \text {. }
$$

Evaluation of Eq. (A1) is now done in such a way that for a given point $\vec{R}_{0}$, of the dual lattice through which runs the cut surface $\Sigma_{\sigma}$, Eq. (A1) is evaluated for all pairs for which $\vec{R}_{0}$, is situated on the connecting line of the constituents of the pair. In Fig. 1 the dual lattice points are represented by crosses. Eq. (A1) can then be written in the form

$$
I_{j}\left(\Sigma_{\sigma}\right)=\sum_{\overrightarrow{\mathrm{m}}>\mathbf{\mathrm { n }}} \Phi_{j, s}^{\overrightarrow{\mathrm{m}}-\overrightarrow{\mathrm{n}}} b_{s}^{\sigma} e^{i \overrightarrow{\mathrm{q}} \cdot \overrightarrow{\mathrm{R}}} \overrightarrow{\mathrm{n}}\left(1-e^{i \overrightarrow{\mathrm{a}} \cdot\left(\overrightarrow{\mathrm{R}}_{0}^{\overrightarrow{\mathrm{m}}}-\overrightarrow{\mathrm{R}}_{0}^{\overrightarrow{\mathrm{n}}}\right)}\right),
$$

where $\vec{R}_{0}^{\mathrm{m}}$ is taken from above $\Sigma_{\sigma}$ and $\vec{R}_{0}^{\vec{n}}$ from below $\Sigma_{\sigma}$. Next we sum over all pairs for which $\overrightarrow{\mathrm{h}}=\overrightarrow{\mathrm{R}}_{0}^{\mathrm{m}}-\overrightarrow{\mathrm{R}}_{0}^{\overrightarrow{\mathrm{n}}}$ is fixed. This yields

$$
\begin{aligned}
I_{j}\left(\Sigma_{\sigma}\right)= & \sum_{R_{0^{\prime}} \in \Sigma_{\sigma}} \sum_{\overrightarrow{\mathrm{h}}}^{\prime} \Phi_{\hat{j}, s^{\mathrm{h}}} b_{s}^{\sigma} e^{i \overrightarrow{\mathrm{a}} \cdot \overrightarrow{\mathrm{R}}_{0^{\prime}}} e^{-i \overrightarrow{\mathrm{a}} \cdot \overrightarrow{\mathrm{h}} / 2}\left(1-e^{i \overrightarrow{\mathrm{a}} \cdot \overrightarrow{\mathrm{h}}}\right) \\
& \times \sum_{h^{\prime}>-|\overrightarrow{\mathrm{h}}| / 2}^{h^{\prime}\langle|\overrightarrow{\mathrm{h}}| / 2} e^{-i \overrightarrow{\mathrm{q}} \cdot \overrightarrow{\mathrm{h}}^{0} h^{\prime}},
\end{aligned}
$$

which $\sum_{\overrightarrow{\mathrm{h}}}^{\prime}$ indicates that only $\overrightarrow{\mathrm{h}}$ 's pointing into the half space above $\Sigma_{\sigma}$ are taken and $\vec{h}^{0}=\vec{h} /|\vec{h}|$. In

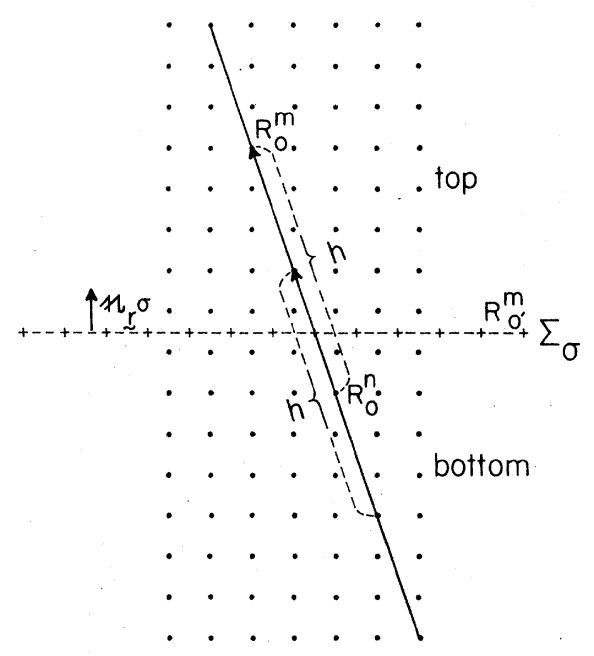

FIG. 1. Two-dimensional cut through cut surface $\Sigma_{\sigma}$ with normal vector $\overrightarrow{\mathrm{n}}_{\mathbf{F}^{\circ}}$. Dual lattice points $R_{0}^{\vec{m}}$ are represented by crosses, ordinary lattice points $R_{0}^{\vec{m}}$ are represented as points. $\vec{h}$ connects ordinary lattice points and points into top half plane. 
Fig. 1 a set of pairs for which $\vec{h}$ is fixed is indicated by brackets and they produce the final multiplicative term of Eq. (A4). It is clear that Eq. (A4) can lead to a qualitative sensible result only if the interaction matrix $\left\{\Phi_{i, k}^{\overrightarrow{\mathrm{h}}}\right\}$ is not so long-ranged that pairs connected over the cut surface $\Sigma_{\sigma}$ in Eq. (A1) connect in fact over additional cut surfaces. In that case Eq. (A2a) has to be modified. This case will, however, not be considered in the following. Eq. (A4) can now be put into the form

$$
\begin{aligned}
I_{j}\left(\Sigma_{\sigma}\right)= & -4 i \sum_{R_{0^{\prime}} \in \Sigma_{\sigma}} \sum_{\overrightarrow{\mathrm{h}}}^{\prime} \Phi_{j_{j}}^{\overrightarrow{\mathrm{h}}} b_{s}^{\sigma} e^{i \overrightarrow{\mathrm{a}} \cdot \overrightarrow{\mathrm{R}}_{0^{\prime}} \sin \left(\overrightarrow{\mathrm{q}} \cdot \frac{1}{2} \overrightarrow{\mathrm{h}}\right)} \\
& \times \sum_{h^{\prime}=0}^{H<|\overrightarrow{\mathrm{h}}|^{/ 2}} \cos \left(\overrightarrow{\mathrm{q}} \cdot \overrightarrow{\mathrm{h}}^{0} h^{\prime}\right) .
\end{aligned}
$$

In the following we are only interested in longwavelength phenomena. Accordingly we can make the approximations

$$
\begin{aligned}
& \sum_{h^{\prime}=0}^{h^{\prime}<|\overrightarrow{\mathrm{h}}|^{\prime 2}} \cos \left(\overrightarrow{\mathrm{q}} \cdot \overrightarrow{\mathrm{h}}^{0} h^{\prime}\right) \sim \frac{1}{2}\left|\overrightarrow{\mathrm{n}}_{\overrightarrow{\mathrm{R}}_{0}^{\prime}} \cdot \overrightarrow{\mathrm{h}}\right|, \\
& \sin \left(\overrightarrow{\mathrm{q}} \cdot \frac{1}{2} \overrightarrow{\mathrm{h}}\right) \sim \frac{1}{2}(\overrightarrow{\mathrm{q}} \cdot \overrightarrow{\mathrm{h}}),
\end{aligned}
$$

where $\vec{n}_{\vec{r}_{0}^{\prime}}$ is the normal vector of the cut surface. Using the Eqs. (A6a) and (A6b) in Eq. (A5) and substituting discrete summation by integration yields

$I_{j}\left(\Sigma_{\sigma}\right) \approx-\frac{i}{2} \sum_{\overrightarrow{\mathrm{h}}} \int_{\Sigma_{\sigma}}\left(d^{2} \overrightarrow{\mathrm{r}}^{\sigma} \cdot \overrightarrow{\mathrm{h}}\right) \Phi_{j, s}^{\overrightarrow{\mathrm{h}}} b_{s}^{\sigma} e^{i \overrightarrow{\mathrm{a}} \cdot \overrightarrow{\mathrm{r}}^{\sigma}}(\overrightarrow{\mathrm{q}} \cdot \overrightarrow{\mathrm{h}})$.

Here use has been made of the fact that $d^{2} \overrightarrow{\mathrm{r}}^{\sigma} \cdot \overrightarrow{\mathrm{h}}$ $>0$ holds for $\vec{h}$ pointing into the half space above $\Sigma_{\sigma}$. The presence of an additional $\vec{h}$ in Eq. (A6b) allows then to write Eq. (A7) as an even function of $\vec{h}$ and dropping the prime on the summation sign. In the presence of a set of cut surfaces $\left\{\Sigma_{0}\right\}$ one obtains instead of Eq. (17)

$$
I_{j}\left(\left\{\Sigma_{\sigma}\right\}\right) \sim-\frac{i}{2} \sum_{\sigma, \overrightarrow{\mathrm{h}}} \int_{\Sigma_{\sigma}}\left(d^{2} \overrightarrow{\mathbf{r}}^{\sigma} \cdot \overrightarrow{\mathrm{h}}\right) \Phi_{j, s}^{\overrightarrow{\mathrm{h}}} b_{s}^{\sigma}(\overrightarrow{\mathrm{q}} \cdot \overrightarrow{\mathrm{h}}) e^{i \overrightarrow{\mathrm{a}^{\circ}} \cdot \overrightarrow{\mathrm{r}}^{\sigma}} .
$$

Let us point out here that the defect sectors of the model Hamiltonian are now characterized by a set of cut surfaces $\left\{\Sigma_{\sigma}\right\}$, where with each cut surface $\Sigma_{\sigma}$ a vector $\vec{b}^{\sigma}$ is associated. By obvious reasons this vector will be called the Burgers vector $\vec{b}^{\sigma}$ of the cut surface.

Equation (A $\left.7^{\prime}\right)$ will now be inserted into Eq. (23) of Sec. III. This yields

$$
\begin{aligned}
\Phi^{\mathrm{int}}(\{\overrightarrow{\mathrm{b}} \overrightarrow{\mathrm{m}}, \overrightarrow{\mathrm{n}}\}) \sim & -\frac{1}{8 N} \sum_{\overrightarrow{\mathrm{q}}}\left(\sum_{\overrightarrow{\mathrm{h}}, \sigma} \int_{\Sigma_{\sigma}}\left(d^{2} \overrightarrow{\mathrm{r}}^{\sigma} \cdot \overrightarrow{\mathrm{h}}\right) \Phi_{j, s}^{\overrightarrow{\mathrm{h}}} b_{s}^{\sigma}(\overrightarrow{\mathrm{q}} \cdot \overrightarrow{\mathrm{h}}) e^{i \overrightarrow{\mathrm{q}} \cdot \overrightarrow{\mathrm{r}}^{\sigma}}\right) \\
& \times \Phi_{j, i^{\prime}}^{-1}(\overrightarrow{\mathrm{q}})\left(\sum_{\overrightarrow{\mathrm{h}}^{\prime}, \sigma^{\prime}} \int_{\Sigma_{\sigma^{\prime}}}\left(d^{2} \overrightarrow{\mathrm{r}}^{\sigma^{\prime}} \cdot \overrightarrow{\mathrm{h}}^{\prime}\right) \Phi_{i^{\prime}, s^{\prime}}^{\overrightarrow{\mathrm{h}}^{\prime}} b_{s^{\prime}}^{\sigma^{\prime}}\left(\overrightarrow{\mathrm{q}} \cdot \overrightarrow{\mathrm{h}}^{\prime}\right) e^{-i \overrightarrow{\mathrm{a}} \cdot \overrightarrow{\mathrm{r}}^{\sigma^{\prime}}}\right)-\frac{1}{4} \sum_{\overrightarrow{\mathrm{m}}, \overrightarrow{\mathrm{n}}} \Phi_{i, k}^{\overrightarrow{\mathrm{m}}-\overrightarrow{\mathrm{n}}} b_{i}^{\overrightarrow{\mathrm{m}}, \overrightarrow{\mathrm{n}}} b_{k}^{\overrightarrow{\mathrm{m}}, \overrightarrow{\mathrm{n}}} .
\end{aligned}
$$

Next we introduce an orthogonal trihedron for fixed $\overrightarrow{\mathrm{q}}$, $\left(\overrightarrow{\mathrm{n}}_{1}(\overrightarrow{\mathrm{q}}), \overrightarrow{\mathrm{n}}_{2}(\overrightarrow{\mathrm{q}}), \overrightarrow{\mathrm{n}}_{3}(\overrightarrow{\mathrm{q}})\right)$, with $\overrightarrow{\mathrm{n}}_{3}(q)=\overrightarrow{\mathrm{q}}^{0}$. With reference to this coordinate system one obtains

$$
I_{j}\left(\left\{\Sigma_{\sigma}\right\}\right)=-\frac{i}{2} \sum_{\overrightarrow{\mathrm{h}}, \sigma} \int_{\Sigma_{\sigma}}\left\{(1 / q)\left(d^{2} \overrightarrow{\mathrm{r}}^{\sigma}\right)_{3}(\overrightarrow{\mathrm{q}} \cdot \overrightarrow{\mathrm{h}})^{2} \Phi_{j, s}^{\overrightarrow{\mathrm{h}}} b_{s}^{\sigma} e^{i \overrightarrow{\mathrm{q}} \cdot \overrightarrow{\mathrm{r}} \sigma}+\left[\left(d^{2} \overrightarrow{\mathrm{r}}^{\sigma}\right)_{1} h_{1}+\left(d^{2} \overrightarrow{\mathrm{r}}^{\sigma}\right)_{2} h_{2}\right] \Phi_{j, s}^{\overrightarrow{\mathrm{h}}} b_{s}^{\sigma}(\overrightarrow{\mathrm{q}} \cdot \overrightarrow{\mathrm{h}}) e^{i \overrightarrow{\mathrm{q}} \cdot \overrightarrow{\mathrm{r}} \sigma}\right\} .
$$

Using Eq. (20) in the form

$$
\Phi_{i, k}(\overrightarrow{\mathrm{q}}) \sim-\frac{1}{2} \sum_{\overrightarrow{\mathrm{h}}} \Phi_{i, k}^{\overrightarrow{\mathrm{h}}}(\overrightarrow{\mathrm{q}} \cdot \overrightarrow{\mathrm{h}})^{2},
$$

one obtains for Eq. (A9)

$$
I_{j}\left(\left\{\Sigma_{\sigma}\right\}\right)=i \sum_{\sigma} \int_{\Sigma_{\sigma}} \frac{\left(d^{2} \overrightarrow{\mathbf{r}}^{\sigma}\right)_{3}}{q} \Phi_{j, s}(\overrightarrow{\mathrm{q}}) b_{s}^{\sigma} e^{i \overrightarrow{\mathrm{q}} \cdot \overrightarrow{\mathrm{r}}^{\sigma}}-\frac{i}{2} \sum_{\overrightarrow{\mathrm{h}}, \sigma} \int_{\Sigma_{\sigma}}\left[\left(d^{2} \overrightarrow{\mathrm{r}}^{\sigma}\right)_{1} h_{1}+\left(d^{2} \overrightarrow{\mathbf{r}}^{\sigma}\right)_{2} h_{2}\right] \Phi_{j, s}^{\overrightarrow{\mathrm{h}}} b_{s}^{\sigma}(\overrightarrow{\mathrm{q}} \cdot \overrightarrow{\mathrm{h}}) e^{i \overrightarrow{\mathrm{q}} \cdot \overrightarrow{\mathrm{r}}^{\sigma}} .
$$

Equation $\left(\mathrm{A} 9^{\prime}\right)$ can be used to write Eq. (A8) in the following form:

$$
\begin{aligned}
& \Phi^{\mathrm{int}}(\{\overrightarrow{\mathrm{b}} \overrightarrow{\mathrm{n}}, \overrightarrow{\mathrm{m}}\})=-\frac{1}{8 N} \sum_{\overrightarrow{\mathrm{q}}}\left[-2 \sum_{\sigma} \int_{\Sigma_{\sigma}} \frac{1}{q}\left(d^{2} \overrightarrow{\mathrm{r}}^{\sigma}\right)_{3} \Phi_{j, s}(\overrightarrow{\mathrm{q}}) b_{s}^{\sigma} e^{i \overrightarrow{\mathrm{q}} \cdot \overrightarrow{\mathrm{r}}^{\sigma}}+\sum_{\sigma} \int_{\Sigma_{\sigma}} \sum_{\overrightarrow{\mathrm{h}}}\left[\left(d^{2} \overrightarrow{\mathrm{r}}^{\sigma}\right)_{1} h_{1}+\left(d^{2} \overrightarrow{\mathrm{r}}^{\sigma}\right)_{2} h_{2}\right] \Phi_{j, s}^{\overrightarrow{\mathrm{h}}} b_{s}^{\sigma}(\overrightarrow{\mathrm{q}} \cdot \overrightarrow{\mathrm{h}}) e^{i \overrightarrow{\mathrm{q}} \cdot \overrightarrow{\mathrm{r}}^{\sigma}}\right. \\
& \times \Phi_{j_{, i}}^{-1},(\overrightarrow{\mathrm{q}})\left(\sum_{\sigma, \vec{h}^{\prime}} \int_{\Sigma_{\sigma}}\left(d^{2} \overrightarrow{\mathrm{r}}^{\sigma}\right)_{3} h_{3}^{\prime} \Phi_{i^{\prime}, s^{\prime}}^{\overrightarrow{\mathrm{h}}^{\prime}} b_{s^{\prime}}^{\sigma}\left(\overrightarrow{\mathrm{q}}^{\circ} h^{\prime}\right) e^{-i \overrightarrow{\mathrm{q}}^{\circ} \cdot \overrightarrow{\mathrm{r}}^{\sigma}}\right) \\
& \left.+\sum_{\sigma, \vec{h}} \int_{\Sigma_{\sigma}}\left[\left(d^{2} \overrightarrow{\mathrm{r}}^{\sigma}\right)_{1} h_{1}+\left(d^{2} \overrightarrow{\mathrm{r}}^{\sigma}\right)_{2} h_{2}\right] \Phi_{i^{\prime}, s^{\prime}}^{\overrightarrow{\mathrm{h}}} b_{s^{\prime}}^{\sigma}(\overrightarrow{\mathrm{q}} \cdot \overrightarrow{\mathrm{h}}) e^{-i \overrightarrow{\mathrm{q}} \cdot \overrightarrow{\mathrm{r}}^{\sigma}}\right]-\frac{1}{4} \sum_{\overrightarrow{\mathrm{m}}, \overrightarrow{\mathrm{n}}} \Phi_{i, k}^{\overrightarrow{\mathrm{m}}-\overrightarrow{\mathrm{n}}} b_{i}^{\overrightarrow{\mathrm{m}}, \overrightarrow{\mathrm{n}}} b_{k}^{\overrightarrow{\mathrm{m}}, \overrightarrow{\mathrm{n}}} .
\end{aligned}
$$


This expression can be evaluated and yields

$$
\begin{aligned}
& \Phi^{\mathrm{int}}\left(\left\{\overrightarrow{\mathrm{b}}^{\overrightarrow{\mathrm{n}}}, \overrightarrow{\mathrm{m}}\right\}\right)=-\frac{1}{8 N} \sum_{\overrightarrow{\mathrm{q}}}\left(\sum_{\sigma, \overrightarrow{\mathrm{h}}} \int_{\Sigma_{\sigma}}\left[\left(d^{2} \overrightarrow{\mathrm{r}}^{\sigma}\right)_{1} h_{1}+\left(d^{2} \overrightarrow{\mathrm{r}}^{\sigma}\right)_{2} h_{2}\right]\right. \\
& \left.\times \Phi_{j, s}^{\overrightarrow{\mathrm{h}}} b_{s}^{\sigma}(\overrightarrow{\mathrm{q}} \cdot \overrightarrow{\mathrm{h}}) e^{i \overrightarrow{\mathrm{q}} \cdot \overrightarrow{\mathrm{r}}^{\sigma} \sigma} \Phi_{j^{-1} i^{\circ}}(\overrightarrow{\mathrm{q}}) \sum_{\sigma^{\prime}, \overrightarrow{\mathrm{h}}^{\prime}} \int_{\Sigma_{\sigma^{\prime}}}\left[\left(d^{2} \overrightarrow{\mathrm{r}}^{\sigma^{\prime}}\right)_{1} h_{1}^{\prime}+\left(d^{2} \overrightarrow{\mathrm{r}}^{\sigma^{\prime}}\right)_{2} h_{2}^{\prime}\right]\left(\overrightarrow{\mathrm{q}} \cdot \overrightarrow{\mathrm{h}}^{\prime}\right) \Phi_{i^{\prime}, s^{\prime}}^{\overrightarrow{\mathrm{h}}^{\prime}} b_{\boldsymbol{s}^{\prime}}^{\sigma^{\prime}} e^{-i \overrightarrow{\mathrm{q}} \cdot \overrightarrow{\mathrm{r}}^{\sigma^{\circ}}}\right)
\end{aligned}
$$

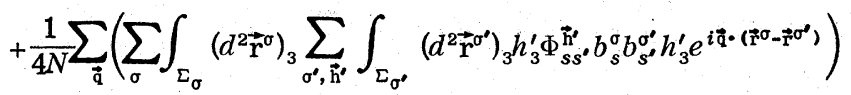

$$
\begin{aligned}
& +\frac{1}{2 N} \sum_{\overrightarrow{\mathrm{q}}}\left(\sum_{\sigma} \int_{\Sigma_{\sigma}}\left(d^{2} \overrightarrow{\mathrm{r}}^{\sigma}\right)_{3} \sum_{\sigma^{\prime}, \overrightarrow{\mathrm{h}}^{\prime}} \int_{\Sigma_{\sigma^{\circ}}}\left[\left(d^{2} \overrightarrow{\mathrm{r}}^{\sigma^{\prime}}\right)_{1} h_{1}^{\prime}+\left(d^{2} \overrightarrow{\mathrm{r}}^{\sigma^{\prime}}\right)_{2} h_{2}^{\prime}\right] \Phi_{s s^{\prime}}^{\overrightarrow{\mathrm{h}}^{\prime}} \cdot b_{s^{\sigma}}^{\sigma} b_{s^{\prime}}^{\sigma^{\prime}}\left(\overrightarrow{\mathrm{q}}^{\mathrm{o}_{0}} \cdot \overrightarrow{\mathrm{h}}^{\prime}\right) e^{i \boldsymbol{q} \cdot\left(\mathbf{r}^{\sigma}-\mathbf{r}^{\sigma^{\prime}}\right)}\right) \\
& -\frac{1}{4} \sum_{\sigma, \overrightarrow{\mathrm{h}}} \int_{\Sigma_{\sigma}}\left(d^{2} \overrightarrow{\mathrm{r}}^{\sigma} \cdot \overrightarrow{\mathrm{h}}\right)\left(\overrightarrow{\mathrm{n}}_{\overrightarrow{\mathrm{r}}^{\sigma}} \cdot \overrightarrow{\mathrm{h}}^{0}\right) \Phi_{i, k}^{\overrightarrow{\mathrm{h}}} b_{i}^{\sigma} b_{k}^{\sigma} \text {. }
\end{aligned}
$$

Here we have expressed the last term of Eq. (A10) in integral form which is derived under the same approximation as Eq. (A7). In order to simplify Eq. (A11) further some formulae from vector algebra will be used. It is rather simple to obtain

$$
\left(d^{2} \overrightarrow{\mathbf{r}}^{\sigma}\right)_{1} h_{1}^{\prime}+\left(d^{2} \overrightarrow{\mathbf{r}}^{\sigma}\right)_{2} h_{2}^{\prime}=\left(d^{2} \overrightarrow{\mathbf{r}}^{\sigma} \times \overrightarrow{\mathrm{q}}^{0}\right) \cdot\left(\overrightarrow{\mathrm{h}}^{\prime} \times q^{0}\right) .
$$

Using

$$
\left(d^{2} \gamma^{\sigma}\right)_{3} h_{3}^{\prime}=\left(d^{2} \overrightarrow{\mathbf{r}}^{\sigma} \cdot \overrightarrow{\mathrm{h}}^{\prime}\right)-\left(d^{2} \overrightarrow{\mathrm{r}}^{\sigma} \times \overrightarrow{\mathrm{q}}^{0}\right) \cdot\left(\overrightarrow{\mathrm{h}}^{\prime} \times q^{0}\right),
$$

we get

$$
\begin{aligned}
\left(d^{2} \overrightarrow{\mathbf{r}}^{\sigma}\right)_{3}\left(d^{2} \overrightarrow{\mathbf{r}}^{\sigma^{\prime}}\right)_{3} h_{3}^{\prime 2}=\left(d^{2} \overrightarrow{\mathbf{r}}^{\sigma} \circ \overrightarrow{\mathbf{h}}^{\prime}\right)\left(d^{2} \overrightarrow{\mathbf{r}}^{\sigma^{\prime} \cdot} \cdot \overrightarrow{\mathbf{h}}^{\prime}\right) & -\left(\overrightarrow{\mathbf{h}}^{\prime} \times \overrightarrow{\mathbf{q}}^{0}\right)\left[\left(d^{2} \overrightarrow{\mathbf{r}}^{\sigma} \times \overrightarrow{\mathbf{q}}^{0}\right)\left(d^{2} \overrightarrow{\mathbf{r}}^{\sigma^{\prime}} \cdot \overrightarrow{\mathbf{h}}^{\prime}\right)+\left(d^{2} \mathbf{r}^{\sigma^{\prime}} \times \overrightarrow{\mathbf{q}}^{0}\right)\left(d^{2} \overrightarrow{\mathbf{r}}^{\sigma} \times \overrightarrow{\mathbf{h}}^{\prime}\right)\right] \\
& +\left(d^{2} \overrightarrow{\mathbf{r}}^{\sigma} \times \overrightarrow{\mathbf{q}}^{0}\right) \cdot\left(\overrightarrow{\mathbf{h}}^{\prime} \cdot \overrightarrow{\mathbf{q}}^{0}\right)\left(d^{2} \overrightarrow{\mathbf{r}}^{\sigma^{\sigma}} \times \overrightarrow{\mathbf{q}}^{0}\right) \cdot\left(\overrightarrow{\mathbf{h}}^{\prime} \times \overrightarrow{\mathbf{q}}^{0}\right) .
\end{aligned}
$$

Use of Eq. (A13) yields

$$
\begin{aligned}
\left(d^{2} \overrightarrow{\mathrm{r}}^{\sigma}\right)_{3} h_{3}^{\prime}\left[\left(d^{2} \overrightarrow{\mathrm{r}}^{0}\right)_{1} h_{1}^{\prime}+\left(d^{2 \overrightarrow{\mathrm{r}}^{\sigma}}\right)_{2} h_{2}^{\prime}\right] & \\
= & \left(d^{2} \overrightarrow{\mathrm{r}}^{\sigma} \times \overrightarrow{\mathrm{q}}^{0}\right) \cdot\left(\overrightarrow{\mathrm{h}}^{\prime} \times \overrightarrow{\mathrm{q}}^{0}\right) \\
& \times\left[\left(d^{2} \overrightarrow{\mathbf{r}}^{\sigma} \cdot \overrightarrow{\mathrm{h}}^{\prime}\right)-\left(d^{2} \overrightarrow{\mathrm{r}}^{\sigma} \times \overrightarrow{\mathrm{q}}^{0}\right) \cdot\left(\overrightarrow{\mathrm{h}}^{\prime} \times \overrightarrow{\mathrm{q}}^{0}\right)\right] .
\end{aligned}
$$

If Eq. (A13) is introduced into the second term of Eq. (A11), the sum over $\vec{q}$ of the first term resulting from Eq. (A13) can immediately be performed yielding the $\delta$ function, i.e.,

$$
\begin{aligned}
& \frac{1}{N} \sum_{\vec{q}} e^{i \vec{q} \cdot\left(\vec{r}^{\sigma}-\vec{r}^{\sigma^{\prime}}\right)} \sim \frac{1}{(2 \pi)^{3}} \int d^{3} q e^{i \vec{q} \cdot\left(\overrightarrow{\mathrm{r}}^{\sigma}-\vec{r}^{\sigma^{\prime}}\right)} \\
& =\delta\left(\overrightarrow{\mathbf{r}}^{\sigma}-\overrightarrow{\mathbf{r}}^{\sigma^{\prime}}\right) \text {. }
\end{aligned}
$$

This term then cancels the last term of Eq. (A11) as can be seen as follows. In order to apply the usual rules for integration over the three-dimensional $\delta$ function the differential of the surface in- tegrals will be written in the form

$$
\begin{aligned}
& \left(d^{2} \overrightarrow{\mathrm{r}}^{\sigma} \cdot \overrightarrow{\mathrm{h}}^{\prime}\right)\left(d^{2} \overrightarrow{\mathrm{r}}^{\sigma^{\prime}} \cdot \overrightarrow{\mathrm{h}}^{\prime}\right) \\
& \quad=\left(d^{2} \overrightarrow{\mathrm{r}}^{\sigma}: \overrightarrow{\mathrm{h}}\right) \operatorname{sgn}\left(\overrightarrow{\mathrm{n}}_{\overrightarrow{\mathrm{r}}^{\sigma^{\circ}}} \cdot \overrightarrow{\mathrm{h}}^{0 \prime}\right) \int_{\sigma \Omega\left(\overrightarrow{\mathrm{r}}^{\sigma^{\circ}}, \overrightarrow{\mathrm{h}}^{\prime}\right)} d^{3} \gamma^{\sigma^{\circ}},
\end{aligned}
$$

where the integral is performed over the volume element

$$
\delta \Omega\left(\overrightarrow{\mathbf{r}}^{\sigma^{\prime}}, \overrightarrow{\mathrm{h}}^{\prime}\right)=\int d^{2} r^{\sigma^{\prime}}\left|\overrightarrow{\mathrm{n}}_{\overrightarrow{\mathbf{r}}^{\sigma^{\prime}}} \cdot \overrightarrow{\mathrm{h}}^{\prime}\right|
$$

and where $\operatorname{sgn}(x)$ indicates the signum function. Using

$$
\int_{\delta \delta\left(\overrightarrow{\mathbf{r}}^{0}, \overrightarrow{\mathrm{r}}^{\prime}\right)} d^{3} r^{\sigma^{\prime}} \delta\left(\overrightarrow{\mathbf{r}}^{\sigma^{\prime}}-\overrightarrow{\mathbf{r}}^{\sigma}\right) f\left(\overrightarrow{\mathbf{r}}^{\sigma^{\prime}}, \overrightarrow{\mathbf{r}}^{\sigma}\right)=f\left(\overrightarrow{\mathbf{r}}^{\sigma}, \overrightarrow{\mathbf{r}}^{\sigma}\right),
$$

the result claimed above follows.

After a trivial algebraic step Eq. (A11) can now be written in the form

$$
\begin{aligned}
& \Phi^{\mathrm{int}}\left(\left\{\overrightarrow{\mathrm{b}^{\mathrm{n}}}, \overrightarrow{\mathrm{m}}\right\}\right)=-\frac{1}{8 N} \sum_{\overrightarrow{\mathrm{q}}}\left(\sum_{\sigma, \overrightarrow{\mathrm{h}}} \int_{\Sigma_{\sigma}}\left(d^{2} \overrightarrow{\mathrm{r}}^{\sigma} \times \overrightarrow{\mathrm{q}}^{0}\right) \cdot\left(\overrightarrow{\mathrm{h}} \times \overrightarrow{\mathrm{q}}^{0}\right) \Phi_{j, s}^{\overrightarrow{\mathrm{h}}} b_{s}^{\sigma}(\overrightarrow{\mathrm{q}} \cdot \overrightarrow{\mathrm{h}}) e^{i \overrightarrow{\mathrm{a}} \cdot \overrightarrow{\mathrm{r}} \sigma}\right) \\
& \times \Phi_{\vec{j}, i^{\prime}}^{-1}(\overrightarrow{\mathrm{q}})\left(\sum_{\sigma^{\prime}, \overrightarrow{\mathrm{h}}^{\prime}} \int_{\Sigma_{\sigma^{\prime}}}\left(d^{2} \overrightarrow{\mathrm{r}}^{\sigma^{\circ}} \times \overrightarrow{\mathrm{q}}^{0}\right) \cdot\left(\overrightarrow{\mathrm{h}}^{\prime} \times \overrightarrow{\mathrm{q}}^{0}\right) \Phi_{i^{\prime}, s^{\prime}}^{\overrightarrow{\mathrm{h}}^{\prime}} b_{s^{\prime}}^{\sigma^{\prime}}\left(\overrightarrow{\mathrm{q}} \cdot \overrightarrow{\mathrm{h}}^{\prime}\right) e^{-i \mathbb{q}^{\prime} \cdot \overrightarrow{\mathrm{r}}^{\sigma^{\prime}}}\right) \\
& -\frac{1}{4 N} \sum_{\overrightarrow{\mathrm{q}}} \sum_{\sigma} \int_{\Sigma_{\sigma}} \sum_{\sigma^{\prime}, \overrightarrow{\mathrm{h}}^{\prime}} \int_{\Sigma_{\sigma^{\prime}}}\left(d^{2} \overrightarrow{\mathrm{r}}^{\sigma} \times \overrightarrow{\mathrm{q}}^{0}\right) \cdot\left(\overrightarrow{\mathrm{h}}^{\prime} \times \overrightarrow{\mathrm{q}}^{0}\right)\left(d^{2} \overrightarrow{\mathrm{r}}^{\sigma^{\prime}} \times \overrightarrow{\mathrm{q}}^{0}\right) \cdot\left(\overrightarrow{\mathrm{h}}^{\prime} \times \overrightarrow{\mathrm{q}}^{0}\right) e^{i \overrightarrow{\mathrm{q}} \cdot\left(\overrightarrow{\mathrm{r}}^{\sigma} \overrightarrow{\mathrm{r}}^{\sigma^{\prime}}\right)} \Phi_{s, s^{\prime}}^{\overrightarrow{\mathrm{h}}} b_{s^{\sigma}}^{\sigma} b_{s^{\prime}}^{\sigma^{\prime}} .
\end{aligned}
$$


This expression is now in a form which allows the application of Stokes' theorem

$$
\int_{\Sigma_{\sigma}} d^{2} \overrightarrow{\mathbf{r}}^{\sigma} \times \vec{\nabla} e^{-i \overrightarrow{\mathrm{d}} \cdot \overrightarrow{\mathrm{r}}^{\sigma}}=\int_{c_{\sigma}} d \overrightarrow{\mathbf{r}}^{\sigma} e^{-i \overrightarrow{\mathrm{a}} \cdot \overrightarrow{\mathbf{r}}^{\sigma}},
$$

where $C_{\sigma}$ is the contour bounding the surface $\Sigma_{\sigma}$. Using Eq. (A17) in Eq. (A16) yields

$$
\begin{aligned}
& \Phi^{\mathrm{int}}\left(\left\{\overrightarrow{\mathrm{b}}^{\overrightarrow{\mathrm{n}}, \overrightarrow{\mathrm{m}}}\right\}\right)=-\frac{1}{8 N} \sum_{\overrightarrow{\mathrm{q}}} \sum_{\sigma, \sigma^{\prime}} \sum_{\overrightarrow{\mathrm{h}}} \int_{C_{\sigma}} d \overrightarrow{\mathrm{r}}^{\sigma} \cdot\left(\overrightarrow{\mathrm{h}} \times \overrightarrow{\mathrm{q}}^{0}\right)\left(\overrightarrow{\mathrm{q}}^{\mathrm{o}} \cdot \overrightarrow{\mathrm{h}}\right) \Phi_{j, s}^{\overrightarrow{\mathrm{h}}} b_{s}^{\sigma} \Phi_{j, i^{\prime}}^{-1}(\overrightarrow{\mathrm{q}}) \\
& \times \sum_{\overrightarrow{\mathrm{h}}^{\prime}} \int_{C_{\sigma^{\prime}}} d \overrightarrow{\mathrm{r}}^{\sigma^{\circ}} \cdot\left(\overrightarrow{\mathrm{h}}^{\prime} \times \overrightarrow{\mathrm{q}}^{0}\right)\left(\overrightarrow{\mathrm{q}}^{0} \circ \overrightarrow{\mathrm{h}}\right) \Phi_{i^{\prime}, s^{\prime}}^{\overrightarrow{\mathrm{h}}^{\prime}} b_{s^{\prime}}^{\sigma^{\prime}}, e^{i \overrightarrow{\mathrm{q}} \cdot\left(\overrightarrow{\mathrm{r}}^{\sigma}-\overrightarrow{\mathrm{r}}^{\sigma^{\prime}}\right)} \\
& -\frac{1}{4 N} \sum_{\overrightarrow{\mathrm{q}}} \sum_{\sigma, \sigma^{\prime}} \sum_{\overrightarrow{\mathrm{h}}^{\prime}} \Phi_{s, s^{\prime}}^{\overrightarrow{\mathrm{h}}^{\circ}} b_{s}^{\sigma} b_{s^{\prime}}^{\sigma^{\prime}} \int_{C_{\sigma}} d \overrightarrow{\mathrm{r}}^{\sigma} \cdot\left(\overrightarrow{\mathrm{h}}^{\prime} \times \overrightarrow{\mathrm{q}}^{0}\right) \int_{C_{\sigma^{\prime}}} d \overrightarrow{\mathbf{r}}^{\sigma^{\circ}} \cdot\left(\overrightarrow{\mathrm{h}}^{\prime} \times \overrightarrow{\mathrm{q}}^{0}\right) \frac{e^{i \overrightarrow{\mathrm{q}} \cdot\left(\overrightarrow{\mathrm{r}}^{\sigma} \overrightarrow{\mathrm{r}}^{\sigma^{\circ}}\right)}}{q^{2}} .
\end{aligned}
$$

Using the quantities defined under Eq. (9) in Sec. II and writing

$$
\overrightarrow{\mathrm{a}} \times \overrightarrow{\mathrm{b}}=\epsilon_{i j k} a_{j} b_{k},
$$

where $\epsilon_{i j k}$ is the Levi-Civita tensor allows us to put Eq. (A18) into the final form given under Eq. (24) in Sec. III.

*Permanent address: Institut für Theoretische Physik, Universität des Saarlandes, 66 Saarbrücken, W. Germany.

${ }^{1} \mathrm{E}$. Kroener, Kontinuum Theorie der Versetzungen (Springer, Berlin, 1958).

${ }^{2}$ A. Seeger, in Encyclopedia of Physics, edited by S. Flügge (Springer, Berlin, 1955), p. 383; Phys. Status Solidi 1, 669 (1961).

${ }^{3}$ J. Friedel, Dislocations (Pergamon, Oxford, 1964).
${ }^{4} \mathrm{~J}$. P. Hirth and J. Lothe, Theory of Dislocations (McGraw-Hill, New York, 1968).

${ }^{5}$ B. K. D. Gairola, Phys. Status Solidi B 85, 577 (1978).

${ }^{6}$ A. Kochendörfer and A. Seeger, Z. Phys. 127, 533 (1950).

${ }^{7}$ A. Holz, J. Phys. Lett. 19, L-331 (1978).

${ }^{8} \mathrm{G}$. Leibfried, in Encyclopedia of Physics, edited by S. Flïgge (Springer, Berlin, 1955), p. 104.

${ }^{9}$ K. Huang, Proc. R. Soc. A 203, 178 (1950). 